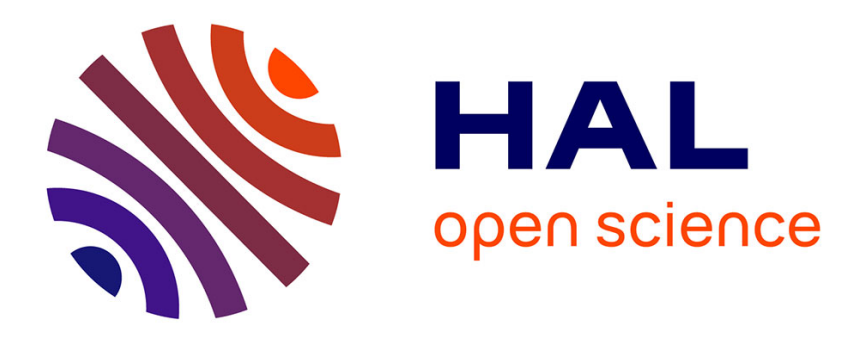

\title{
Polynomial Chaos expansion for subsurface flows with uncertain soil parameters
}

Pierre Sochala, O. Le Maitre

\section{To cite this version:}

Pierre Sochala, O. Le Maitre. Polynomial Chaos expansion for subsurface flows with uncertain soil parameters. Advances in Water Resources, 2013, 62, pp.139-154. 10.1016/j.advwatres.2013.10.003 . hal-00931639

\section{HAL Id: hal-00931639 \\ https://hal.science/hal-00931639}

Submitted on 17 Jan 2014

HAL is a multi-disciplinary open access archive for the deposit and dissemination of scientific research documents, whether they are published or not. The documents may come from teaching and research institutions in France or abroad, or from public or private research centers.
L'archive ouverte pluridisciplinaire HAL, est destinée au dépôt et à la diffusion de documents scientifiques de niveau recherche, publiés ou non, émanant des établissements d'enseignement et de recherche français ou étrangers, des laboratoires publics ou privés. 


\title{
Polynomial Chaos expansion for subsurface flows with uncertain soil parameters
}

\author{
P. Sochala ${ }^{\mathrm{a}, *}$ O.P. Le Maître ${ }^{\mathrm{b}}$ \\ ${ }^{a} B R G M, 3$ avenue Claude Guillemin, 45060 Orléans, France \\ ${ }^{b}$ LIMSI-CNRS, 91403 Orsay, France, and Duke University, MEMS Dept. Durham, NC, USA.
}

\begin{abstract}
The effects of uncertainty in hydrological laws are studied on subsurface flows modeled by Richards' equation. The empirical parameters of the water content and the hydraulic conductivity are considered as uncertain inputs of the model. One-dimensional infiltration problems are treated and the influence of the variability of the input parameters on the position and the spreading of the wetting front is evaluated. A Polynomial Chaos (PC) expansion is used to represent the output quantities and permits to significantly reduce the number of simulations in comparison with a classical Monte-Carlo method. A non-intrusive spectral projection supplies the coefficients of the PC decomposition. Three test cases with different hydrological laws are presented and demonstrate that second order PC expansions are sufficient to represent our quantities of interest owing to smooth dependences for the considered problems. Our results show a correlation between the position and the spreading of the wetting front and an amplification of the input uncertainty for all models. For each test case, five configurations with variable initial saturation state are investigated. The global sensitivity analysis indicates that the relative influence of an input parameter changes according to the output quantity considered and the initial saturation of the soil. The impact of the assumed distributions for the parameters is also briefly illustrated.
\end{abstract}

Keywords: uncertainty quantification, global sensitivity analysis, Polynomial Chaos expansions, Richards' equation, non-intrusive spectral decomposition

\section{Introduction}

Water infiltration in soil is one of the key processes in hydrology and have significant influence in many situations, such as for the determination of water table positions, the prediction of floods, and the quantification of erosion risks. The more general model to describe airwater flows in soil is a two-phase flow system which can be simplified into the Richards' equation (Richards (1931) [44]) in non-deformable porous medium with constant air pressure. This equation may be written in two formulations depending on the selected unknown, the hydraulic head $(\psi)$ or the water content $(\theta)$. In this work, we consider the formulation based on the hydraulic head which can be used for saturated soil contrary to the water content formulation. Two constitutive relations characterizing the soil hydrological behavior are needed to solve the Richards' equation: the water content (or the capillary capacity when the non-conservative form is used) and the hydraulic conductivity are functions of the hydraulic head. The most classical constitutive relations were proposed by Brooks and Corey (1964) [12] and Van Genuchten (1980) 51]; other models were also proposed in Gardner (1958) 22], Haverkamp et al. (1977) 26], Vogel et

\footnotetext{
* Corresponding author

Email addresses: p.sochala@brgm.fr (P. Sochala), olm@limsi.fr (O.P. Le Maître)
}

al. (2001) [52] and Assouline and Tartakovsky (2001) [5]. The four parameters for the water content are the residual and the saturated water contents, a scaling factor related to the air entry pressure head and an exponent related to the pore-size distribution. Those for the conductivity are the conductivity at saturation, a scaling factor generally equal to this used in the water content and an exponent often depending of the exponent of the water content. These parameters can be either directly measured, fitted to experimental data or determined from pedotransfer functions. However these parameters are seldom exactly known, justifying a stochastic description, due to space heterogeneities, measurement inaccuracies and the ill-posedness of inverse problems inherent in parameter estimation techniques (Kool et al. 1987) 32].

Several studies are devoted to uncertainty quantification in subsurface flows. A common approach is to derive deterministic equations for the mean and the variance of the pressure (and the water content). For instance, Mantoglou and Gelhar [37] propose a mean model representation for large-scale unsaturated flows. Tartakovsky et al. treat the conductivity at saturation as a random field in the steady case [49] and consider uncertain parameters as random fields in the transient case [50]. Lu and Zhang [36] study hydraulic properties with multimodal distribution. Some authors have investigated how progresses the wetting front with uncertain hydraulic properties: Bresler 
and Dagan (1983) [1] and Protopapas and Bras (1990) 42 in a single layer soil, Dillah and Protopapas (2000) [18] in layered soils. Other types of study in a stochastic context have also been done: Dostert et al. [19] consider uncertain saturated conductivity for inverse problems and Woodward et al. [54] realize a local sensitivity analysis with correlated parameters, among others. The approach based on the first two moments of pressure is unadapted to risk managment which requires a determination of complete pdfs (for example, to estimate the probability of occurence of rare events). To this aim, Wang and Tartakovsky [53] obtain infiltration-rate pdfs by employing simplified flows. Forster 21] consider a Polynomial Chaos expansions (PCE) of the Richards equation with uncertain permeability. In this work, we extend these studies to single layer soils with uncertain hydraulic properties. While previous studies used the Monte-Carlo (MC) method to estimate the mean and the variance of the pressure head (and the water content), we propose to construct PCE (Ghanem and Spanos 1991) 23] for the position and the spreading of the wetting front. The main interest in using $\mathrm{PCE}$, compared to MC methods, is that few input configurations are sufficient to determine the expansion. In addition, a complete characterization of the model outputs, including the mean, the variance and the sensitivity indices, is directly derived from the expansion. High-order moments and pdf's can also be estimated using the PCE by a classical sampling of the set of random events related to the inputs.

The MC method is widely used in uncertainty quantification. The principle of this method is to generate a random sample set of events, to evaluate the model for each element of this sample set, and to compute estimates of moments, sensitivity indices or any statistical quantity of interest from the set of model outputs. Although its robustness, the application of the MC method to models having high CPU time is often prohibitive as a large sample set is necessary to achieve well converged estimates. Several strategies are possible to overcome this difficulty. A first way consists in reducing the number of simulations by using more efficient sampling methods, e.g. Quasi Monte-Carlo methods (Moskowitz and Caflish 1996) 39] and Latin hypercube sampling (Helton and Davis 2003) [27]. Alternatively, to reduce the computational cost of the MC method one can use models based on simplified physics. For instance, the Green and Ampt model (1911) 24] is a simplified form of the Darcy's law, valid only for the water infiltration in soil, and the infiltration transfer functions can be used to replace the full Richards' equation (Besbes and De Marsily 1984) [9]. Some methods can even rely on physics-free modeling techniques, through the construction of meta-models, such as for the polynomial regression and Gaussian process modeling (Sacks et al. 1989) [45]. One has then to trade-off the construction cost of the meta-model with its predictive capacity. In this work, we use PCE because of its construction which require a fairly low number of full-model simulations, whenever the model outputs are sufficiently smooth with respect to the inputs. Furthermore, the PCE furnishes a compact and convenient representation of the outputs, containing all the information on their variability and dependences with the inputs. For example, sensitivity indices are readily obtained from the PCE of the outputs (Crestaux et al. 2009). Different approaches are possible to compute the PCE (Le Maitre and Knio, 2010) 35], and we rely here on the Non Intrusive Spectral Projection (NISP, Reagan et al, 2003) 43]. The main advantage of the NISP is to reuse the deterministic code as a "black box". We apply the NISP methodology to evaluate the effect of stochastic hydrological laws on subsurface flows. The parameters of the water content and the conductivity are modeled as uniform and independent random variables and the position and the spreading of the wetting front are the output quantities of interest. We investigate three test cases (TC) with Haverkamp's, Van Genuchten's and Brooks-Corey's relations.

The objective of this work is twofold. The first one is to apply the NISP method for obtaining a reliable metamodel demanding a low number of simulations. Several tests are performed to assess the quality and accuracy of the NISP-PCE through careful MC validations. The second objective is to demonstrate the efficiency of the PCE to conduct a priori global sensitivity analysis. To this end, the influence of uncertain parameters is investigated on three different test cases with several soil saturation states. Regarding the methodology, the essential result concerns the efficiency of the second order PCE to represent the model outputs, the first moments and the pdf's deduced from the PCE being the same as those estimated via classical MC sampling. From the physical standpoint, the sensitivity analysis evidences a significant correlation between the position and the spreading of the wetting front. Moreover, the uncertainty on the parameters is shown to have a large and non trivial impact on the shape and position of the wetting front. In particular, the degree of saturation of the soil is shown to significantly affect the wetting front position. The three models also amplify the uncertainty, the coefficients of variation of the outputs being greater than those of the inputs. A global sensitivity analysis (Sobol 1993 [47], Homma and Saltelli 1996 [28]) is employed to hierarchize the respective influences of the uncertain parameters, which depend on the TC (physical model), the soil saturation state and the assumed parameter distributions.

This paper is organized as follows. Section 2 introduces the Richards' equation and the constitutive relations. The numerical method employed in a deterministic configuration is described. Section 3 concerns the treatment of uncertainty: the description of the stochastic modeling for the uncertain inputs parameters, the construction of the PCE for the model outputs and the characterization of its variability (sensitivity analysis). Section 4 details the results for the three different TC with constitutive relations presented in section 2. Finally, Section 5 summarizes the main findings of this work and provides recommendations 
for the application of the proposed methodology to more complex hydrological models.

\section{The framework}

\subsection{Physical model}

We are interested in a simplified model of air and water subsurface flows in which the water is incompressible and the air pressure is constant. The flow is described by the Richards' equation which is obtained by combining the mass water conservation equation and the Darcy's law and whose the $1 \mathrm{D}$ conservative form is

$$
\begin{cases}\partial_{t}(\theta(\psi))-\partial_{x}\left(K(\psi)\left(\partial_{x} \psi+1\right)\right)=0 & \text { in }] 0, L[\times] 0, T], \\ \psi(x=0, \cdot)=\psi_{\mathrm{t}} \text { and } \psi(x=L, \cdot)=\psi_{\mathrm{b}} & \text { on }] 0, T], \\ \psi(\cdot, t=0)=\psi^{0} & \text { on }] 0, L[,\end{cases}
$$

where $\psi(x, t)[m]$ is the hydraulic head, $\theta(\psi)[-]$ the volumetric water content, $K(\psi)\left[m \cdot s^{-1}\right]$ the hydraulic conductivity and $x[m]$ the vertical coordinate. Let $] 0, L[\subset \mathbb{R}$ be the physical bounded domain and $T$ the final time of the simulation. The top and the bottom boundaries conditions are respectively denoted by $\psi_{\mathrm{t}}$ and $\psi_{\mathrm{b}}$ and the initial condition is $\psi^{0}$. In our cases, water infiltration is generated by the top condition but a volumetric water source or sink term $\left[s^{-1}\right]$ could be added on the right-hand side of the equation.

Two constitutive laws $\psi \mapsto \theta(\psi)$ and $\psi \mapsto K(\psi)$ are necessary to close the model. To correctly describe the hydrological behavior of a soil, these two relations contain some parameters fitted to experimental measurements. Here, we focus on the most commonly used relations suggested by Haverkamp, Van Genuchten and Brooks and Corey. In the following, $\theta_{\mathrm{r}}$ and $\theta_{\mathrm{s}}$ denote respectively the residual and the saturated water contents and $K_{\mathrm{s}}$ is the saturated conductivity. The Haverkamp's relations (HR) are

$$
\theta(\psi)=\frac{\theta_{s}-\theta_{r}}{1+|\alpha \psi|^{\beta}}+\theta_{r}, \quad K(\psi)=\frac{K_{s}}{1+|A \psi|^{\gamma}} .
$$

The scaling factor $\alpha$ is the inverse of the hydraulic head when the effective saturation $\tilde{\theta}$ (defined as $\left(\theta-\theta_{\mathrm{r}}\right)\left(\theta_{\mathrm{S}}-\right.$ $\left.\theta_{\mathrm{r}}\right)^{-1}$ ) is equal to one-half. The scaling factor $A$ is the inverse of the hydraulic head when the conductivity is half of the saturated conductivity. The exponents $\beta$ and $\gamma$ are the slopes of the log-log plots of $\left(\tilde{\theta}^{-1}-1\right)(\psi)$ and $\left(K_{\mathrm{s}} K^{-1}-1\right)(\psi)$. The Van Genuchten's relations (VGR) are

$$
\begin{gathered}
\theta(\psi)=\frac{\left(\theta_{\mathrm{s}}-\theta_{\mathrm{r}}\right)}{\left(1+(\epsilon|\psi|)^{\beta}\right)^{\gamma}}+\theta_{\mathrm{r}} \\
K(\psi)=K_{\mathrm{s}} \frac{\left(1-(\epsilon|\psi|)^{\beta-1}\left(1+(\epsilon|\psi|)^{\beta}\right)^{-\gamma}\right)^{2}}{\left(1+(\epsilon|\psi|)^{\beta}\right)^{\frac{\gamma}{2}}} .
\end{gathered}
$$

The scaling factor $\epsilon$ is related to the inverse of the air entry pressure, the exponent $\beta$ is a measure of the poresize distribution and the exponent $\gamma$ is defined as $1-\beta^{-1}$.
The Brooks-Corey 's relations (BCR) are

$$
\begin{gathered}
\theta(\psi)= \begin{cases}\theta_{\mathrm{s}}\left(\frac{\psi}{\psi_{\mathrm{a}}}\right)^{-\frac{1}{b}} & \text { if } \psi<\psi_{\mathrm{a}} \\
\theta_{\mathrm{s}} & \text { if } \psi \geq \psi_{\mathrm{a}}\end{cases} \\
K(\psi)= \begin{cases}K_{\mathrm{s}}\left(\frac{\psi}{\psi_{\mathrm{a}}}\right)^{-\gamma} & \text { if } \psi<\psi_{\mathrm{a}} \\
K_{\mathrm{s}} & \text { if } \psi \geq \psi_{\mathrm{a}}\end{cases}
\end{gathered}
$$

The scaling factor $\psi_{\mathrm{a}}$ is the air entry pressure head, the exponent $b$ is related to the pore-size distribution and the exponent $\gamma$ is defined as $2+3 b^{-1}$.

In this work, we consider uncertain parameters except the saturated and the residual water content which can be easily obtained experimentally (Van Genuchten 1980 [51]). For each hydrological law, we define the vector $\boldsymbol{d}$ associated to empirical and independent parameters and whose the stochastic model is specified in subsection 3.1

$$
\begin{array}{ll}
\boldsymbol{d}=\left(\alpha, \beta, K_{\mathrm{s}}, A, \gamma\right) & \text { for HR, } \\
\boldsymbol{d}=\left(K_{\mathrm{s}}, \epsilon, \beta\right) & \text { for VGR, } \\
\boldsymbol{d}=\left(K_{\mathrm{s}}, \psi_{\mathrm{a}}, \gamma\right) & \text { for BCR. }
\end{array}
$$

\subsection{Deterministic discretization}

A discontinuous Galerkin (DG) method is chosen for the space discretization of Richards' equation because this type of method ensure local conservation as finite volumes (Manzini and Ferraris 2004) [38 and mixed finite elements (Knabner and Schneid 2002) 31] and have highorder accuracy as finite elements (Celia and Bouloutas 1990) [14] and mixed finite elements. Several DG methods can be applied for Richards' equation and two-phase flows in porous media especially the Local Discontinous Galerkin (LDG) method (Fagherazzi et al. 2004 [20], Bastian et al. 2007 [8]) and the non-symmetric or the symmetric interior penalty Galerkin method (Klieber and Rivière 2006 [30], Bastian 2002 [7], Sochala et al. 2009 [48]). In the present work, we prefer the LDG method whose the fluxes are linear. Concerning time discretization, the usual schemes employed with DG methods are explicit RungeKutta (Cockburn and Shu 1998) [15] or diagonally implicit (Bastian 2002) [7]. We propose instead to use backward differentiation formula (BDF, Curtiss and Hirschfelder 1952) [17] since this type of scheme have high-order accuracy, avoid the CFL condition particularly restrictive for explicit schemes when diffusion term is present, and is more efficient than implicit Runge-Kutta schemes for problems where the nonlinear solver is expensive.

To approximate the Richards' equation, we consider the following mixed formulation which has the same fluxes that those used for the Laplacian,

$$
\left\{\begin{array}{l}
K(\psi)^{-1} v(\psi)=-\left(\partial_{x} \psi+1\right) \\
\partial_{t}(\theta(\psi))+\partial_{x} v(\psi)=0
\end{array}\right.
$$

A formulation depending only on the primal variable $\psi$ is possible by a local elimination of the variable $v$ (see 
Arnold et al. 2001 [4] for the bilinear form of the Laplacian) and can be concisely written as the following semidiscrete form,

$$
\int_{\tau} \partial_{t}\left(\theta\left(\psi_{h}\right)\right) \varphi+a_{\tau}\left(\psi_{h}, \varphi\right)=b_{\tau}\left(\psi_{h}, \varphi\right)
$$

where $\psi_{h}$ is the space approximation of $\psi, \varphi$ is a test function, the form $a_{\tau}$ represents the diffusive part and the form $b_{\tau}$ collects the contributions of the gravity and the boundaries conditions. In this work, the degree of the piecewise functions $\psi_{h}$ and $\varphi$ is equal to one. The unsteady term is approximated by the second order BDF,

$$
\begin{aligned}
\frac{3}{2 \delta t} \int_{\tau} \theta\left(\psi_{h}^{n}\right) \varphi+a_{\tau}\left(\psi_{h}^{n}, \varphi\right) & \simeq b_{\tau}\left(\psi_{h}^{n}, \varphi\right) \\
& +\frac{1}{\delta t} \int_{\tau}\left(2 \theta\left(\psi_{h}^{n-1}\right)-\frac{1}{2} \theta\left(\psi_{h}^{n-2}\right)\right) \varphi
\end{aligned}
$$

where $\psi_{h}^{n}$ denotes the value taken by $\psi_{h}$ at time $n \delta t$. This nonlinear equation is solved by a quasi-Newton method. The discrete functions $\psi_{h}^{n-1}$ and $\psi_{h}^{n-2}$ being known, successive approximations $\psi_{h}^{n, m}$ of $\psi_{h}^{n}$ are computed from

$$
\begin{aligned}
\frac{3}{2 \delta t} \int_{\tau}\left(\theta\left(\psi_{h}^{n, m}\right)\right. & \left.+\partial_{\psi} \theta\left(\psi_{h}^{n, m}\right)\left(\psi_{h}^{n, m+1}-\psi_{h}^{n, m}\right)\right) \varphi \\
+\widehat{a}_{\tau}\left(\psi_{h}^{n, m}\right. & \left., \psi_{h}^{n, m+1}, \varphi\right)=b_{\tau}\left(\psi_{h}^{n, m}, \varphi\right) \\
& +\frac{1}{\delta t} \int_{\tau}\left(2 \theta\left(\psi_{h}^{n-1}\right)-\frac{1}{2} \theta\left(\psi_{h}^{n-2}\right)\right) \varphi
\end{aligned}
$$

The further argument added to the form $a_{\tau}$ refers to the following linearization $\int_{\tau} K\left(\psi_{h}^{n, m}\right)^{-1} v_{h}^{n, m+1} \varphi$. For the first time step, the Crank-Nicolson scheme is used. The convergence criterion imposed that the relative Euclidean norm of the component vector associated with $\delta \psi_{h}^{n, m}$ is lower than a user-defined tolerance (typically $10^{-4}$ ). The CPU time for one simulation of our TC is several minutes.

\section{Uncertainty quantification}

\subsection{Uncertainty model and PC expansion}

We focus on the uncertainty propagation due to the uncertain empirical parameters of the water content and the conductivity. Each uncertain parameter in the constitutive relations is modeled as a random variable, such that following the notations of subsection 2.1 the input vector $\boldsymbol{d}$ of size $N(N=5$ for $\mathrm{HR}$ and $N=3$ for VGR and BCR) is random and defined on an abstract probability space $\mathcal{P}:=(\Omega, \Sigma, d \mu)$, where $\Omega$ is the set of random events, $\Sigma$ a sigma-algebra and $d \mu$ a probability measure. We denote $\boldsymbol{d}(\omega)$ a realization of the random inputs. In order to perform the global sensitivity analysis of the models with respect to the uncertain parameter, we assume that the components $\boldsymbol{d}_{i}$, for $i=1, \ldots, N$, are mutually independent random variables, each with uniform distribution and equal coefficient of variation. Specifically, $\boldsymbol{d}_{i}(\omega)$ has a uniform distribution with unit probability to fall in the range $\overline{d_{i}}(1 \pm 0.1)$, where $\overline{d_{i}}$ is the expected value of $\boldsymbol{d}_{i}$ :

$$
\mathbb{E}\left[\boldsymbol{d}_{i}\right]=\int_{\Omega} \boldsymbol{d}_{i}(\omega) d \mu(\omega):=\overline{d_{i}} .
$$

In view of computing the PCE expansion of the model outputs, the input vector is expressed in terms of $N$ independent standard random variables $\xi_{i}$ all having uniform distribution in the unit interval:

$$
\boldsymbol{d}_{i}(\omega)=\overline{d_{i}}\left(0.9+0.2 \xi_{i}(\omega)\right), \quad \xi_{i} \sim \mathcal{U}[0,1] .
$$

With a slight abuse of notation, we shall denote hereafter $\boldsymbol{d}(\boldsymbol{\xi}), \boldsymbol{\xi}=\left(\xi_{1}, \ldots, \xi_{N}\right)$, the random vector of parameters. Since the $\xi_{i}$ are independent, their joint probability density function factorizes to

$$
\begin{gathered}
p_{\boldsymbol{\xi}}\left(\xi_{1}, \ldots, \xi_{N}\right)=\prod_{i=1}^{N} p_{\mathcal{U}[0,1]}\left(\xi_{i}\right), \\
p_{\mathcal{U}[0,1]}(\xi)= \begin{cases}1 & \xi \in[0,1], \\
0 & \text { otherwise. }\end{cases}
\end{gathered}
$$

Let us denote $y$ a model output. Because the Richards' model is deterministic for given realization of the input vector $\boldsymbol{d}(\boldsymbol{\xi})$ the output is seen as functional of $\boldsymbol{\xi}$, denoted $y(\boldsymbol{\xi})$. We assume that the random output $y$ is a secondorder random variable,

$$
\mathbb{E}\left[y^{2}\right]=\int_{\Xi} y(\boldsymbol{\xi})^{2} p_{\boldsymbol{\xi}}(\boldsymbol{\xi}) d \boldsymbol{\xi}<+\infty
$$

where $\Xi:=[0,1]^{N}$. The PCE of $y(\boldsymbol{\xi})$, truncated at order $p$ is written as

$$
y(\boldsymbol{\xi}) \approx \widehat{y}(\boldsymbol{\xi}):=\sum_{k \in \mathcal{K}(p)} y_{k} \phi_{k}(\boldsymbol{\xi})
$$

where $k \in \mathbb{N}^{N}$ is a multi-index, $\mathcal{K}(p)$ the set of multiindexes for order $p,\left\{y_{k}\right\}$ is the set of deterministic PC coefficients (or spectral modes) of $y$ and the random functionals $\phi_{k}$ are orthogonal polynomials such that

$\mathbb{E}\left[\phi_{k} \phi_{l}\right]=:\left\langle\phi_{k}, \phi_{l}\right\rangle=\int_{\Xi} \phi_{k}(\boldsymbol{\xi}) \phi_{l}(\boldsymbol{\xi}) p_{\boldsymbol{\xi}}(\boldsymbol{\xi}) d \boldsymbol{\xi}=\delta_{k l}\left\langle\phi_{k}, \phi_{k}\right\rangle$.

For the total degree truncation, the multi-index set is defined as

$$
\mathcal{K}(p)=\left\{k \in \mathbb{N}^{N}, \sum_{i=1}^{N} k_{i} \leq p\right\}
$$

The Cameron and Martin theorem [13] states the convergence of the PCE in the mean-squared sense for $p \rightarrow \infty$. The set $\left\{\phi_{k}, k \in \mathcal{K}(p)\right\}$ forms the PC basis whose dimension $P+1$ is related to $N$ and truncature order $p$ through $P+1=\operatorname{card} \mathcal{K}=(p+N) ! /(p ! N !)$. In fact, the stochastic polynomials $\phi_{k}$ are simply products of one-dimensional Legendre polynomials $\mathcal{L}_{j}$ defined on the unit interval:

$$
\phi_{k}(\boldsymbol{\xi})=\prod_{i=1}^{N} \mathcal{L}_{k_{i}}\left(\xi_{i}\right), \quad k \in \mathcal{K}(p) .
$$


Finally, we observe that the previous PCE can be constructed for other types of distributions for the $\boldsymbol{\xi}$, provided its components remain independent, leading to other families of polynomials, see e.g. (Xiu and Karniadakis 2002 [55]) and (Le Maître and Knio, 2010 [35]). Also, the random vector $\boldsymbol{d}$ may have a distribution different than for $\boldsymbol{\xi}$, by introducing a non-linear transformation $\boldsymbol{\xi} \mapsto \boldsymbol{d}(\boldsymbol{\xi})$. For example, to deal with log-normal distributions for the saturated hydraulic conductivity, as in Dillah and Protopapas (2000) [18], one would use standard normal Gaussian $\xi$ and an exponential transformation.

\subsection{Non intrusive spectral projection}

Different means can be use to determine the PC-coefficient $y_{k}$ in the PCE of $y(\boldsymbol{\xi})$. In this paper, we rely on the non intrusive spectral projection (NISP) method, (see Reagan, 2003 [43], Le Maître and Knio, 2010 35]) which corresponds to a definition of $\hat{y}$ as the $L_{2}$-orthogonal projection of $y(\boldsymbol{\xi})$ on the subspace spanned by the $\left\{\phi_{k}\right\}$. One advantage of this approach is that it is non-intrusive, in the sense that deterministic codes can be reused as black-box without any adaptation. This feature is particularly appealing here because of the non-linearities of the Richards' equation that would require specific treatments for the implementation of an intrusive stochastic Galerkin method.

NISP exploits the orthogonality of the basis. Indeed

$$
\begin{aligned}
y(\boldsymbol{\xi})=\sum_{k \in \mathbb{N}^{N}} y_{k} \phi_{k}(\boldsymbol{\xi}) \Rightarrow\left\langle y, \phi_{k}\right\rangle & =\sum_{i \in \mathbb{N}^{N}} y_{i}\left\langle\phi_{i}, \phi_{k}\right\rangle \\
& =y_{k}\left\langle\phi_{k}, \phi_{k}\right\rangle, \forall k \in \mathbb{N}^{N} .
\end{aligned}
$$

The normalization factor $\left\langle\phi_{k}, \phi_{k}\right\rangle$ corresponds to the squared norm of the polynomial $\phi_{k}$ and is known analytically from the definition of the Legendre polynomials (Abramowitz and Stegun, 1970) [1]. Therefore, the determination of the coefficients $y_{k}$ amounts the evaluation of $N$-dimensional integrals which can be approximated by numerical cubature,

$$
\left\langle y, \phi_{k}\right\rangle=\int_{\Xi} y(\boldsymbol{\xi}) \phi_{k}(\boldsymbol{\xi}) p_{\boldsymbol{\xi}}(\boldsymbol{\xi}) d \boldsymbol{\xi} \simeq \sum_{j=1}^{N_{p}} w_{j} y\left(\boldsymbol{x}_{j}\right) \phi_{k}\left(\boldsymbol{x}_{j}\right),
$$

where the $N_{p}$ integration points $\boldsymbol{x}_{j}$ and associated weights $w_{j}$ define the cubature rule. Since the integration is $N$ dimensional, with $N$ potentially large, full-tensorization of one-dimensional quadrature rules (e.g. Gauss' quadratures) are prohibitively costly and sparse cubature rules, based on Smolyak's formula (see Appendix A), are used in this work. The number of cubature points then depends on the level of the cubature formula, which in turn yields a formula which integrate exactly polynomial up to a certain degree. Therefore, the level of the cubature formula for the numerical integration depends both on the truncature order selected for the PCE and on the quantity of interest $y$. A minimal requirement for the selection of the cubature level is that it verify the discrete orthogonality condition

$$
\sum_{j=1}^{N_{p}} w_{j} \phi_{i}\left(\boldsymbol{x}_{j}\right) \phi_{k}\left(\boldsymbol{x}_{j}\right)=\left\langle\phi_{i}, \phi_{k}\right\rangle \delta_{i, k}, \quad \forall i, k \in \mathcal{K}(p) .
$$

However, complex quantities of interest with non-polynomial dependences may require richer cubature rules, i.e. higher level, to accurately compute the correlations between $y$ and the set of $\phi_{i}$ in its approximation.

\subsection{Statistic estimators}

A particularly attractive feature of the PCE is the easiness to recover information and characterize the variability, in particular the mean, the variance and the Sobol indices of a quantity of interest. We adopt the common convention that the zero order polynomial, equal to 1 , is denoted $\phi_{0}$. Thus, the mean $\mu(y)$ of $y$ is simply the coefficient $y_{0}$,

$$
\mu(y)=\langle y, 1\rangle=\sum_{k \in \mathcal{K}\left({ }^{p}\right)} y_{k}\left\langle\phi_{k}, \phi_{0}\right\rangle \quad \Rightarrow \quad \mu(y)=y_{0} .
$$

Likewise, using the orthogonality of the polynomials, the variance $\sigma_{\mathrm{PC}}^{2}(y)$ is estimated from its PCE approximation through

$$
\begin{aligned}
\sigma^{2}(y) & =\left\langle\left(y-y_{0}\right),\left(y-y_{0}\right)\right\rangle \approx\left\langle\left(\widehat{y}-y_{0}\right),\left(\widehat{y}-y_{0}\right)\right\rangle \\
& =\left\langle\left(\sum_{k \in \mathcal{K}(p) \backslash 0} y_{k} \phi_{k}\right),\left(\sum_{l \in \mathcal{K}(p) \backslash 0} y_{l} \phi_{l}\right)\right\rangle \\
& =\sum_{k, l \in \mathcal{K}(p) \backslash 0} y_{k} y_{l}\left\langle\phi_{k}, \phi_{l}\right\rangle \\
& =\sum_{k \in \mathcal{K}(p) \backslash 0} y_{k}^{2}\left\langle\phi_{k}, \phi_{k}\right\rangle .
\end{aligned}
$$

The Sobol indices, which express the part of the variability due to each input parameter (or group of input parameters), are obtained immediately from the PCE (Crestaux et al. 2009 [16]). In particular, the first order sensitivity index for the input parameter associated to $\xi_{i}$, denoted $S_{i}$, is the conditional variance $\sigma_{i}^{2}(\widehat{y})$ divided by the total variance $\sigma^{2}(\widehat{y})$,

$$
S_{i}=\frac{\sigma_{i}^{2}(\widehat{y})}{\sigma^{2}(\widehat{y})}, \quad \text { with } \quad \sigma_{i}^{2}(\widehat{y})=\sum_{k \in K_{i}} y_{k}^{2}\left\langle\phi_{k}, \phi_{k}\right\rangle,
$$

where $K_{i}$ is the set of the multi-indices such that

$$
K_{i}:=\left\{k \in \mathcal{K}(p), k_{j}=\left\{\begin{array}{ll}
>0, & j=i \\
0, & j \neq i
\end{array}\right\} .\right.
$$

To estimate the pdf or high-order moments of the PCE of $y(\boldsymbol{\xi})$, we use MC method for generating a sample set of quantity of interest, $Y_{\mathrm{PC}}=\left\{\widehat{y}^{(1)}, \ldots, \widehat{y}^{(M)}\right\}$ where $M$ is the sample set dimension and $\widehat{y}^{(i)}$ denotes the value of $\widehat{y}$ at samples $\boldsymbol{\xi}^{(i)}$ drawn at random. We stress that to 
generate $Y_{\mathrm{PC}}$ one needs not to solve the original model, but simply to evaluate the PCE $\widehat{y}(\boldsymbol{\xi})$ at the $\boldsymbol{\xi}^{(i)}$. The pdf of $\widehat{y}$ is then estimated using the Gaussian kernel density estimator (Bowman and Azzalini 1997 [10]),

$$
p_{\eta}(y)=\frac{1}{M \eta} \sum_{m=1}^{M} G\left(\frac{y-\widehat{y}^{(m)}}{\eta}\right), \quad G(x)=\frac{1}{\sqrt{2 \pi}} e^{\left(-\frac{x^{2}}{2}\right)} .
$$

The parameter $\eta$ must be fittingly chosen to avoid spurious oscillations (if $\eta$ is too small) and excessive smoothing (if $\eta$ is too large).

For the purpose of $\mathrm{PCE}$ validation, we shall also compare moments and pdf's based on the PCE with those determined by classical MC method. Using the same samples $\boldsymbol{\xi}^{(i)}$, the corresponding MC sample set of quantity of interest is $Y_{\mathrm{MC}}=\left\{y^{(1)}, \ldots, y^{(M)}\right\}$, whose generation requires solving $M$ times the Richards' equation. In particular, the PCE and MC sample sets will be used to estimate the normalized $l^{2}$-error denoted $e$ and defined as

$$
e=\mathbb{E}\left[(y-\widehat{y})^{2}\right] / \mathbb{E}\left[y^{2}\right] .
$$

The MC estimator of this error is

$$
e^{2} \approx \frac{\sum_{i=1}^{m}\left(y^{(i)}-\widehat{y}^{(i)}\right)^{2}}{\sum_{i=1}^{m}\left(y^{(i)}\right)^{2}}
$$

\section{Results}

We consider 1D downward infiltration problems with hydrological laws presented in subsection 2.1. We focus on the position and the spreading of the wetting front as the quantities of interest in the analysis. The wetting front position $x_{\mathrm{f}}$ is defined as the location of the half-sum value $\psi_{\mathrm{f}}$ of the minimal and maximal hydraulic head in the domain. For the present simulations, the hydraulic head varying monotonically (almost surely) in space, such that these extreme values are observed at the boundaries and, using our notations, it comes

$$
x_{\mathrm{f}}:=\psi_{h}^{-1}\left(\psi_{\mathrm{f}}\right), \quad \psi_{\mathrm{f}}=\frac{1}{2}\left(\psi_{\mathrm{b}}+\psi_{\mathrm{t}}\right) .
$$

Similarly, the spreading of the wetting front, $s_{\mathrm{f}}$, is uniquely defined as the spatial extend of the domain where the hydraulic head takes value in the centered $90 \%$ range of variation. It has for expression

$$
s_{\mathrm{f}}=\left|\psi_{h}^{-1}\left(\psi_{0.05}\right)-\psi_{h}^{-1}\left(\psi_{0.95}\right)\right|, \quad \psi_{k}=\psi_{\mathrm{b}}+k\left(\psi_{\mathrm{t}}-\psi_{\mathrm{b}}\right),
$$

$k=0.05$ or 0.95 . The two quantities of interest are schematically illustrated in Fig. 1. Furthermore, the position mostly characterizes water advection process, while the spreading is related to water diffusion process in the soil.

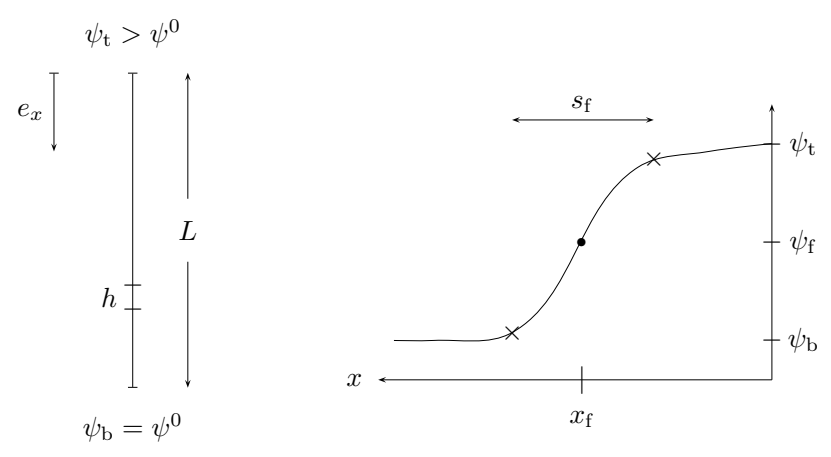

Figure 1: left: Basic notations; right: quantities of interest of the wetting front.

\subsection{Test case 1}

\subsubsection{Test case description}

The first TC was proposed by Haverkamp et al. (1977) [26]. The soil consists of sand and has properties modeled by (11),

$$
\begin{gathered}
\theta(\psi, \boldsymbol{d}(\boldsymbol{\xi}))=\frac{\theta_{s}-\theta_{r}}{1+\left|\alpha\left(\xi_{1}\right) \psi\right|^{\beta\left(\xi_{2}\right)}}+\theta_{r} \\
K(\psi, \boldsymbol{d}(\boldsymbol{\xi}))=\frac{K_{\mathrm{s}}\left(\xi_{3}\right)}{1+\left|A\left(\xi_{4}\right) \psi\right|^{\gamma\left(\xi_{5}\right)}},
\end{gathered}
$$

with deterministic parameters $\theta_{s}=0.287$ and $\theta_{r}=0.075$. The vector $\boldsymbol{d}=\left(\alpha, \beta, K_{\mathrm{s}}, A, \gamma\right)$ of the random parameters has components $\boldsymbol{d}_{i}$ having a uniform distribution $\boldsymbol{d}_{i}\left(\xi_{i}\right)=\overline{d_{i}}\left(0.9+0.2 \xi_{i}\right), \xi_{i} \sim \mathcal{U}[0,1], 1 \leq i \leq 5$, with respective mean values $\bar{\alpha}=0.0271 \mathrm{~cm}^{-1}, \bar{\beta}=3.96, \overline{K_{\mathrm{s}}}=$ $9.44 \cdot 10^{-3} \mathrm{~cm} . \mathrm{s}^{-1}, \bar{A}=0.0524 \mathrm{~cm}^{-1}$ and $\bar{\gamma}=4.74$. It corresponds to the ranges of variations for the water content parameters

$$
\alpha \in 10^{-2} \cdot[2.44,2.98], \quad \beta \in[3.56,4.36],
$$

and for the conductivity parameters

$$
\begin{gathered}
K_{\mathrm{s}} \in 10^{-3} \cdot[8.47,10.38], \quad A \in 10^{-2} \cdot[4.72,5.76], \\
\gamma \in[4.27,5.21] .
\end{gathered}
$$

The coefficient of variation $c_{v}(=\sigma / \mu)$ of this probability distribution is equal to $5.8 \%$. The resulting variability in the water content and conductivity can be appreciated from Fig. 2, which depicts them as functions of the hydraulic head. The black lines correspond to the models for the mean value of $\boldsymbol{d}$, while the gray shadow corresponds to the models for 500 random realizations of $\boldsymbol{d}$. The length of the domain is $L=80 \mathrm{~cm}$, the final time is $T=600 \mathrm{~s}$, and the boundary and initial conditions are $\psi_{\mathrm{b}}=-61.5 \mathrm{~cm}$, $\psi_{\mathrm{t}}=-20.7 \mathrm{~cm}$, and $\psi^{0}(x)=\psi_{\mathrm{b}}$. Preliminary simulations have shown satisfying convergence of the deterministic discrete approximation for a mesh size $h=0.25 \mathrm{~cm}$ and a time steps $\delta t=12 s$. These discretization parameters are kept fixed in subsequent simulations. 

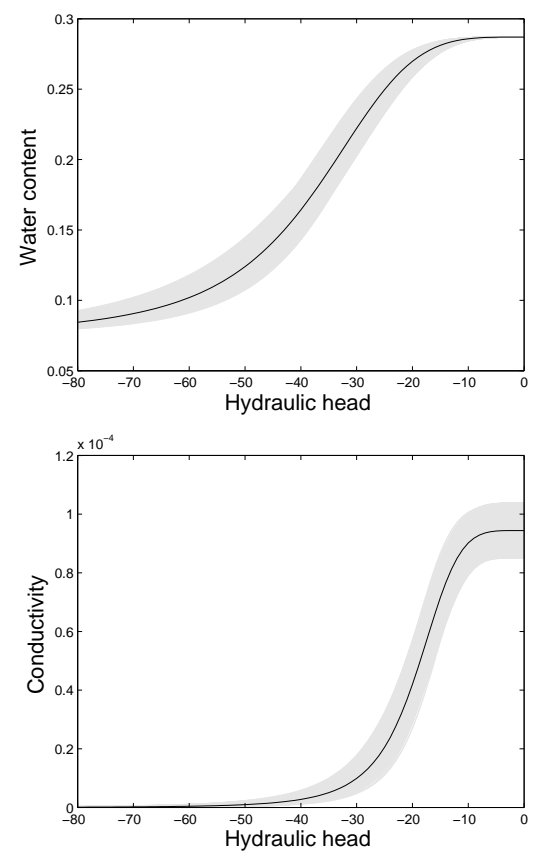

Figure 2: TC1 - Soil properties obtained for $\overline{\boldsymbol{d}}$ (black) and 500 random samples of $\boldsymbol{d}$ (gray).

\subsubsection{Validation of $P C E$ and first moments analysis}

First moments (mean $\mu$, standard deviation $\sigma$ and skewness $s$ ) computed by MC using three sample sets of increasing dimension and PCE up to $\mathbb{P}_{3}$ are reported in Tab. 1 . The number of simulations $N_{\text {sim }}$ corresponds to the sample sets dimension $M$ for the MC method and the number of integration points $N_{p}$ for the PCE (see subsection 3.2). In the PCE computation, the projection uses the cubature formula with minimal level to ensure the orthogonality condition for the considered PC order. We observe that the two approaches agree for sufficiently large sample set dimension (MC) and PCE order. However, it is seen that $\mathrm{MC}$ requires a much larger number of simulations. Fig. 3 presents the MC estimate of the mean and the standard deviation of the position with \pm 3 bootstrap error. A minimum of $10^{4} \mathrm{MC}$ realizations is necessary to consider the convergence (the number of simulations is identical for the spreading). On the contrary, PCE requires less than 200 simulations. Polynomial degrees equal to 2 or 3 permit to finely obtain the first three moments. The normalized $l^{2}$-error $e$ (see subsection 3.3) is also provided in the table, showing that $\mathrm{PC}-\mathbb{P}_{2}$ yields an error $<1 \%$ on the quantities of interest.

In Fig. 4a and 4b, we compare the pdf's of $x_{\mathrm{f}}$ and $s_{\mathrm{f}}$ using the MC method and $\mathrm{PC}-\mathbb{P}_{1}$ : the difference between the curves is due to the non linear dependences neglected in $\mathrm{PC}-\mathbb{P}_{1}$. We also observe the symmetric character of the pdf's, which explain the zero skewness reported for $\mathrm{PC}-\mathbb{P}_{1}$. For PC $-\mathbb{P}_{2}$, the pdf's in Fig. $4 \mathrm{c}$ and $4 \mathrm{~d}$ compare well with the $\mathrm{MC}$ estimates, confirming the results of Tab. 1. Considering these results, the PC - $\mathbb{P}_{2}$ is used

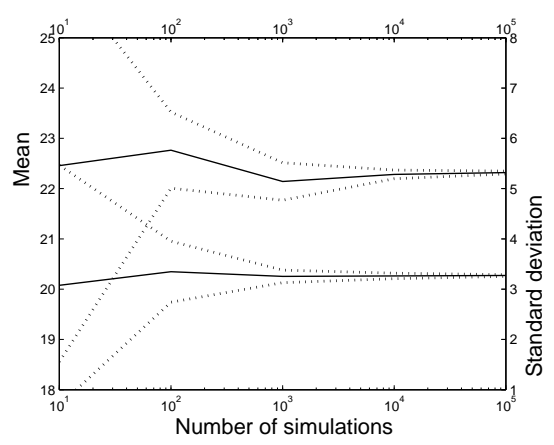

Figure 3: TC1 - Convergence of the MC estimate of $\mu\left(x_{\mathrm{f}}\right)$ and $\sigma\left(x_{\mathrm{f}}\right)$ with \pm 3 bootstrap error.

in subsequent analyses of this section. We recall that this expansion requires 51 simulations only.

Table 1: TC1 - moments and normalized error of the quantities of interest $x_{\mathrm{f}}$ and $s_{\mathrm{f}}$.

\begin{tabular}{cccccccccc}
\hline & \multicolumn{1}{c}{$x_{\mathrm{f}}$} & & & & $s_{\mathrm{f}}$ & & & \\
& $N_{\text {sim }}$ & $\mu$ & $\sigma$ & $s$ & $e$ & $\mu$ & $\sigma$ & $s$ & $e$ \\
& - & $c m$ & $c m$ & $c m$ & $\%$ & $c m$ & $c m$ & $c m$ & $\%$ \\
\hline $\mathrm{MC}$ & $10^{3}$ & 22.19 & 3.27 & 0.21 & & 15.02 & 3.13 & 0.50 & \\
$\mathrm{MC}$ & $10^{4}$ & 22.29 & 3.27 & 0.20 & & 15.14 & 3.10 & 0.46 & \\
$\mathrm{MC}$ & $10^{5}$ & 22.32 & 3.27 & 0.19 & & 15.14 & 3.10 & 0.48 & \\
\hline $\mathrm{PC}-\mathbb{P}_{1}$ & 11 & 22.34 & 3.23 & 0.00 & 1.30 & 15.15 & 3.03 & 0.00 & 2.89 \\
$\mathrm{PC}-\mathbb{P}_{2}$ & 51 & 22.33 & 3.27 & 0.20 & 0.14 & 15.14 & 3.10 & 0.47 & 0.37 \\
$\mathrm{PC}-\mathbb{P}_{3}$ & 151 & 22.34 & 3.27 & 0.19 & 0.09 & 15.15 & 3.10 & 0.48 & 0.07 \\
\hline
\end{tabular}

Fig. 5 presents $10^{4}$ realizations of the $\mathrm{PC}-\mathbb{P}_{2}$ couples $\left(x_{\mathrm{f}}, s_{\mathrm{f}}\right)$ with three isolines of the joint pdf. An interesting observation is that the position and the spreading are rather well-correlated. Indeed, the correlation coefficient $r$, defined as the covariance of the two variables divided by the product of their standard deviation, is equal to 0.81 meaning that $66 \%$ of the dispersion of the scatter is due to a linear relation between the two output quantities. Moreover, the slope of the regression line (0.78) is positive, denoting an increasing front spreading as the front displacement velocity increases. This is illustrated on the top plot of Fig. 6 where the profiles of the pressure corresponding to the extreme values of $x_{\mathrm{f}}$ (over the PCE sample sets) are represented. The profiles corresponding to the extreme values of $s_{\mathrm{f}}$ are almost identical to those for the extreme $x_{\mathrm{f}}$, owing to the strong correlation. The three isolines of the joint pdf localize iso-probabilistic events associated to the first decile, the median and the last decile. This plot highlights the skewed character of the joint distribution, with longer tails toward larger values.

\subsubsection{Effect of soil saturation}

To study the effect of the soil saturation state on the wetting front, we consider five couples $\left(\psi_{\mathrm{b}}, \psi_{\mathrm{t}}\right)$ with constant difference $\psi_{\mathrm{b}}-\psi_{\mathrm{t}}=-40.8 \mathrm{~cm}$. We recall that the initial condition is $\psi^{0}(x)=\psi_{\mathrm{b}}$. The computations are 


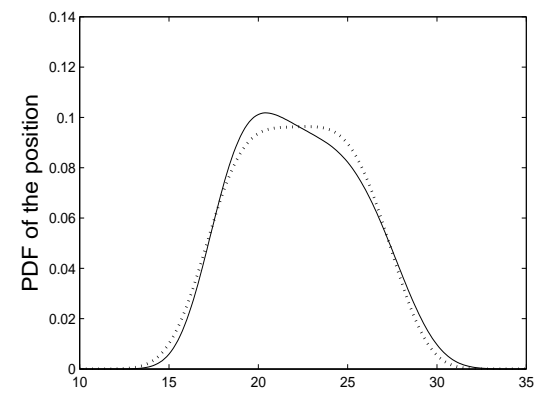

(a) $p_{1}\left(x_{\mathrm{f}}\right)$ estimated by $\mathrm{MC}$ and $\mathrm{PC}-\mathbb{P}_{1}$

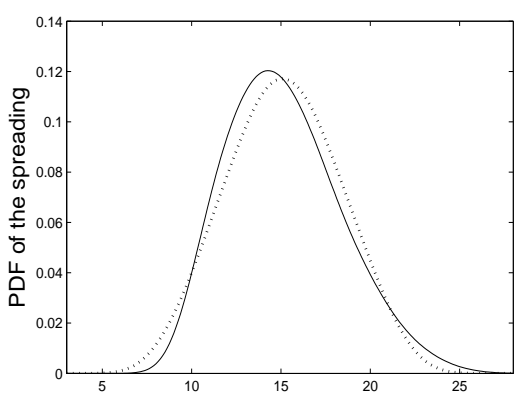

(b) $p_{1}\left(s_{\mathrm{f}}\right)$ estimated by $\mathrm{MC}$ and $\mathrm{PC}-\mathbb{P}_{1}$

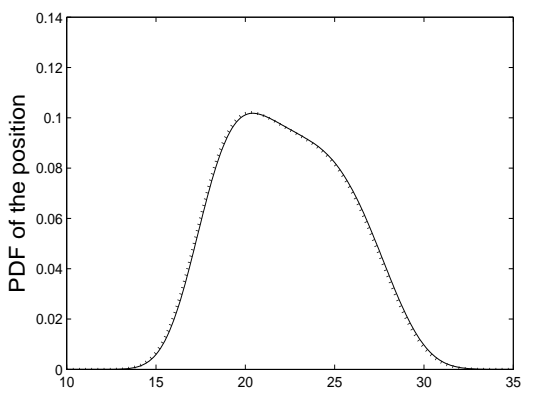

(c) $p_{1}\left(x_{\mathrm{f}}\right)$ estimated by $\mathrm{MC}$ and $\mathrm{PC}-\mathbb{P}_{2}$

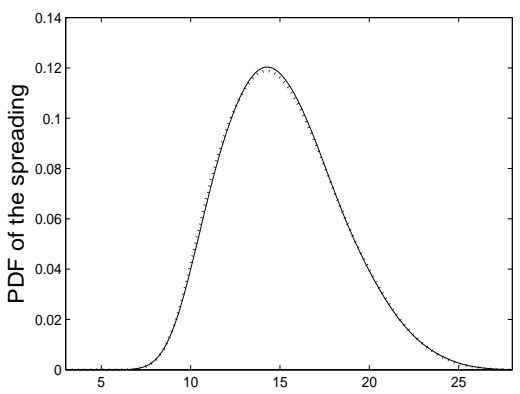

(d) $p_{1}\left(s_{\mathrm{f}}\right)$ estimated by $\mathrm{MC}$ and $\mathrm{PC}-\mathbb{P}_{2}$

Figure 4: TC1 - pdf's of $x_{\mathrm{f}}$ and $s_{\mathrm{f}}$ obtained with MC (solid line) and PCE (dotted line), $\eta=1$.

performed with PC - $\mathbb{P}_{2}$. Tab. 2 reports the statistic estimators for each configuration. The coefficient of determination $R^{2}$ (equal to the square of the correlation coefficient $r$ ) is also reported. As expected, the increasing of the top and initial conditions augments the mean of the
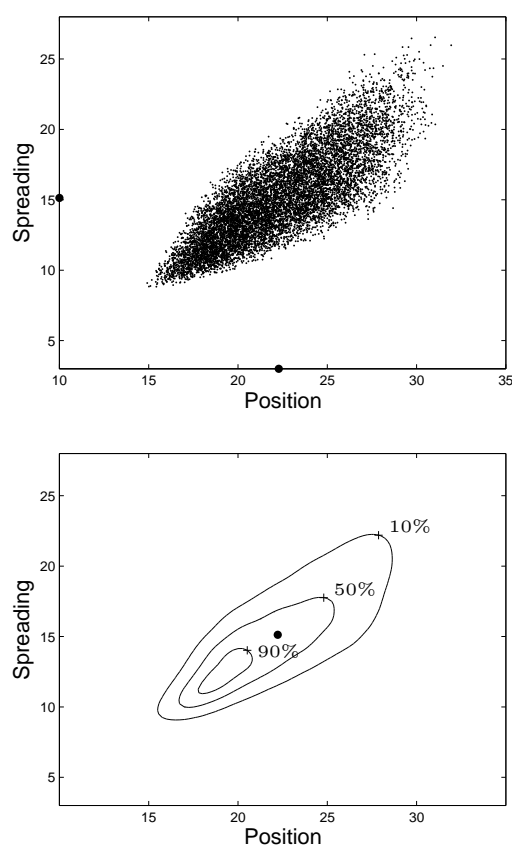

Figure 5: TC1 - top: $10^{4} \mathrm{PC}-\mathbb{P}_{2}$ realizations of $\left(x_{\mathrm{f}}, s_{\mathrm{f}}\right)$; bottom: isolines of the joint pdf.

position because the water velocity is proportional to the conductivity according to the Darcy's law. Here, the mean value of the spreading also rises since the increasing of the saturation accentuates the diffusion process. Indeed, the modification of the pressure profile is illustrated on the bottom plot of Fig. [6 where the hydraulic head for $\overline{\boldsymbol{d}}$ is plotted for each values of the boundary conditions. The mean front position and spreading raise with the saturation. However, the variation of each output is essentially the same for all the saturation states. Also, the model amplify the uncertainty since the coefficient of variation of the outputs are greater than those of the inputs. At last, the coefficients of determination reveal that a significant part of the dispersion (60\% on average) is explained by a linear relation.

The first order sensitivity indices are plotted on Fig. 7 In the following, the index $S_{p}^{q}\left(\psi_{\mathrm{r}}\right)$ corresponds to the parameter $p$ and concerns the quantity of interest $q$ when the top condition is $\psi_{\mathrm{r}}$. We first observe that for all the saturation states, the sum of the first order indices is close to 1 , meaning low interactions between parameters and an essentially additive model for the quantities of interest. For the position, the exponents $\beta$ and $\gamma$ have no influence. When the saturation is increased, the more influent parameter is successively $A, K_{\mathrm{S}}$ and $\alpha$ with $S_{A}^{x_{\mathrm{f}}}(-20.7)=0.84, S_{K_{\mathrm{s}}}^{x_{\mathrm{f}}}(-10)=0.40$ and $S_{\alpha}^{x_{\mathrm{f}}}(0)=0.60$. So, the front displacement is governed by $\alpha$ when the top of the column is close to the saturation $\left(\psi_{\mathrm{t}} \geq-5 \mathrm{~cm}\right)$ and by $A$ when the soil is far from saturation $\left(\psi_{\mathrm{t}} \leq-15 \mathrm{~cm}\right)$. For the spreading, the parameter $A$ is mainly determinant, except for the more saturated configuration for which 

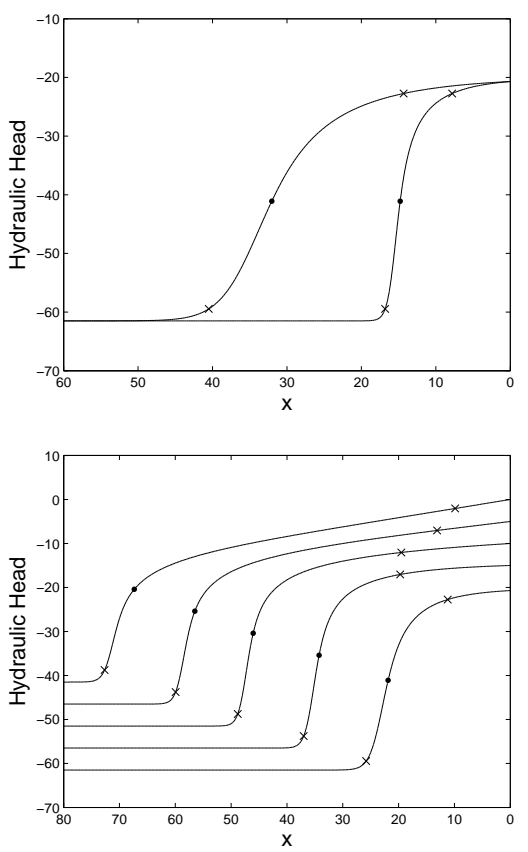

Figure 6: TC1 - top: Pressure corresponding to the extreme values of $x_{\mathrm{f}}$; bottom: Pressure obtained with $\overline{\boldsymbol{d}}$ for the five saturation states reported in Tab 2.

$S_{\alpha}^{s_{\mathrm{f}}}(0)=0.46$ is the highest index. To summarize, the parameter $A$ is the more determinant far from saturation, while $\alpha$ is overtaking close to saturation. At intermediate saturation, $K_{\mathrm{s}}$ is also seen to be significant, although being dominant (for the front position) in only one configuration.

Table 2: TC1 - Moments of the quantities of interest using $\mathrm{PC}-\mathbb{P}_{2}$ for five saturation states.

\begin{tabular}{ccccccccc}
\hline & & $x_{\mathrm{f}}$ & \multicolumn{1}{c}{$s_{\mathrm{f}}$} & & & \\
$\psi^{0}$ & $\psi_{\mathrm{t}}$ & $\mu$ & $\sigma$ & $c_{v}$ & $\mu$ & $\sigma$ & $c_{v}$ & $R^{2}$ \\
$c m$ & $c m$ & $c m$ & $c m$ & $\%$ & $c m$ & $c m$ & $\%$ & $\%$ \\
\hline-61.5 & -20.7 & 22.33 & 3.27 & 15 & 15.14 & 3.10 & 20 & 66 \\
-55.8 & -15 & 34.84 & 3.33 & 10 & 18.49 & 3.82 & 21 & 58 \\
-50.8 & -10 & 46.82 & 3.23 & 7 & 30.33 & 4.55 & 15 & 43 \\
-45.8 & -5 & 57.61 & 4.01 & 7 & 48.02 & 4.04 & 8 & 61 \\
-40.8 & 0 & 68.73 & 5.12 & 7 & 64.05 & 3.87 & 6 & 74 \\
\hline
\end{tabular}

\subsection{Test case 2}

\subsubsection{Test case description}

The second TC was proposed by Celia and Bouloutas (1990) 14]. The soil corresponds to a field in New Mexico with properties modeled by (2),

$$
\theta(\psi, \boldsymbol{d}(\boldsymbol{\xi}))=\frac{\left(\theta_{\mathrm{s}}-\theta_{\mathrm{r}}\right)}{\left(1+\zeta^{\beta\left(\xi_{3}\right)}\right)^{\gamma}}+\theta_{\mathrm{r}},
$$

and

$$
K(\psi, \boldsymbol{d}(\boldsymbol{\xi}))=K_{\mathrm{s}}\left(\xi_{1}\right) \frac{\left(1-\zeta^{\beta\left(\xi_{3}\right)-1}\left(1+\zeta^{\beta\left(\xi_{3}\right)}\right)^{-\gamma}\right)^{2}}{\left(1+\zeta^{\beta\left(\xi_{3}\right)}\right)^{\frac{\gamma}{2}}},
$$
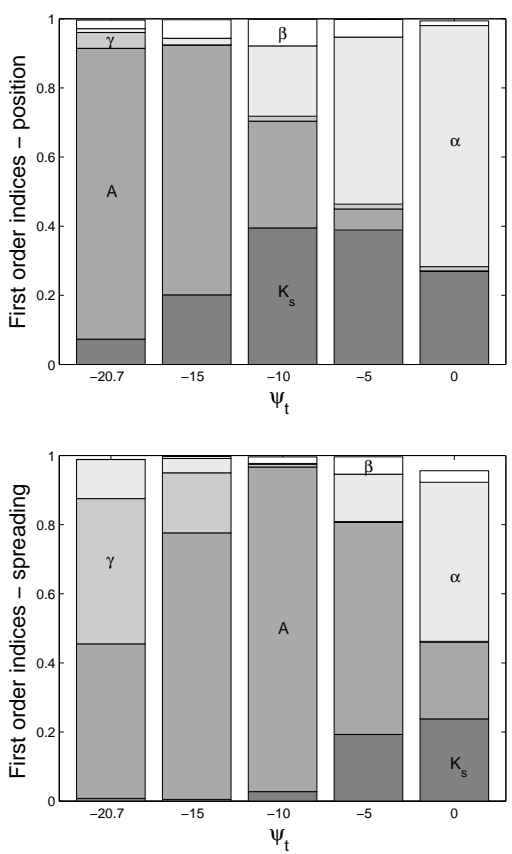

Figure 7: TC1 - First order sensitivity indices using PC $\mathbb{P}_{2}$ for five saturation states.

where $\zeta=\epsilon\left(\xi_{2}\right)|\psi|$. The deterministic parameters are $\theta_{\mathrm{s}}=$ 0.368 and $\theta_{r}=0.102$. The random parameters vector $\boldsymbol{d}=\left(K_{\mathrm{s}}, \epsilon, \beta\right)$ has components $\boldsymbol{d}_{i}$ uniformly distributed with $\pm 10 \%$ range and respective mean values $\overline{K_{\mathrm{s}}}=9.22$. $10^{-3} \mathrm{~cm} \mathrm{~s}^{-1}, \bar{\epsilon}=0.0335 \mathrm{~cm}^{-1}, \bar{\beta}=2$ and $\bar{\gamma}=1-\bar{\beta}^{-1}$. It corresponds to the ranges of variations

$K_{\mathrm{s}} \in 10^{-3} \cdot[8.30,10.14], \quad \epsilon \in[0.03,0.037], \quad \beta \in[1.8,2.2]$.

The resulting variability in the water content and conductivity can be appreciated from Fig. 8, which depicts them as functions of the hydraulic head. The length of the domain is $L=2 m$, the final time is $T=1 h$, and the boundary and initial conditions are $\psi_{\mathrm{b}}=-10 \mathrm{~m}, \psi_{\mathrm{t}}=$ $-75 \mathrm{~cm}$, and $\psi^{0}(x)=\psi_{\mathrm{b}}$. Preliminary simulations have shown satisfying convergence of the deterministic discrete approximation for a mesh size $h=0.1 \mathrm{~cm}$ and a time steps $\delta t=1 \mathrm{~min}$. These discretization parameters are kept fixed in subsequent simulations.

\subsubsection{Validation of PCE and first moments analysis}

First moments computed by $\mathrm{MC}$ using three sample sets of increasing dimension and PCE up to $\mathbb{P}_{3}$ are reported in Tab. 3. We observe that the two approaches agree for sufficiently large sample set dimension (MC) and $\mathrm{PCE}$ order. Moreover, MC requires a much larger number of simulations because a minimum of $10^{3} \mathrm{MC}$ realizations is necessary to consider the convergence, while PCE requires less than 40 simulations. As for TC1, the PCE requires a polynomial degree equal to 2 or 3 to correctly estimate the first three moments. The reported normalized 

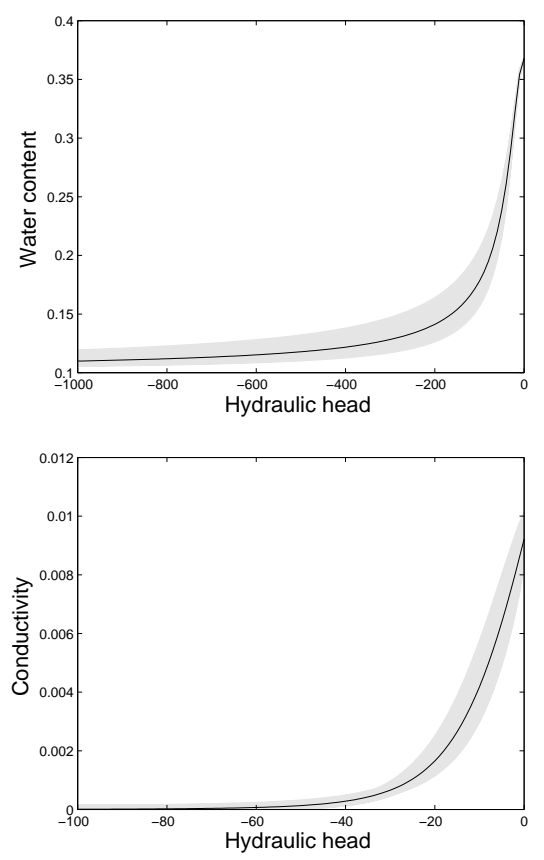

Figure 8: TC2 - Soil properties obtained for $\overline{\boldsymbol{d}}$ (black) and 500 random samples of $\boldsymbol{d}$ (gray).

$l^{2}$-error $e$ indicates that contrary to TC1, a larger cubature formula level is required to ensure decay of $e$, due to cubature errors. It is however observed that the first moments are not significantly affected by this integration error. Further numerical experiments (not shown) have demonstrated that using two additional levels was enough to recover monotonic decay of the $l^{2}$-error, though without noticeable changes in the computed first moments. Concerning the pdf's, not represented here, the findings are identical to TC1: PC - $\mathbb{P}_{1}$ and MC pdf's are close and the difference due to the non linear dependences is reduced with $\mathrm{PC}-\mathbb{P}_{2}$, which requires 19 simulations only and is used in the remainder of this section.

Table 3: TC2 - moments and normalized error of the quantities of interest $x_{\mathrm{f}}$ and $s_{\mathrm{f}}$.

\begin{tabular}{cccccccccc}
\hline & \multicolumn{3}{c}{$x_{\mathrm{f}}$} & & & \multicolumn{1}{c}{$s_{\mathrm{f}}$} & & & \\
& $N_{\text {sim }}$ & $\mu$ & $\sigma$ & $s$ & $e$ & $\mu$ & $\sigma$ & $s$ & $e$ \\
& - & $c m$ & $c m$ & $c m$ & $\%$ & $c m$ & $c m$ & $c m$ & $\%$ \\
\hline $\mathrm{MC}$ & $10^{2}$ & 10.09 & 1.20 & -0.07 & & 3.03 & 0.59 & -0.16 & \\
$\mathrm{MC}$ & $10^{3}$ & 9.81 & 1.20 & 0.15 & & 2.95 & 0.60 & 0.07 & \\
$\mathrm{MC}$ & $10^{4}$ & 9.86 & 1.20 & 0.16 & & 2.96 & 0.60 & 0.09 & \\
\hline $\mathrm{PC}-\mathbb{P}_{1}$ & 7 & 9.87 & 1.20 & 0.00 & 0.82 & 2.98 & 0.58 & 0.00 & 1.84 \\
$\mathrm{PC}-\mathbb{P}_{2}$ & 19 & 9.87 & 1.20 & 0.18 & 0.31 & 2.96 & 0.61 & 0.16 & 1.49 \\
$\mathrm{PC}-\mathbb{P}_{3}$ & 39 & 9.87 & 1.21 & 0.16 & 0.31 & 2.98 & 0.60 & 0.03 & 2.02 \\
\hline
\end{tabular}

Fig. 9 presents $10^{4}$ realizations of the $\mathrm{PC}-\mathbb{P}_{2}$ couples $\left(x_{\mathrm{f}}, s_{\mathrm{f}}\right)$ with three isolines of the joint pdf and shows a better correlation between the position and the spreading than for the previous test case. Here, the correlation coef- ficient is equal to 0.91 implying that $83 \%$ of the dispersion of the scatter is caused by a linear relation between $x_{\mathrm{f}}$ and $s_{\mathrm{f}}$. The slope of the regression line $(0.45)$ is smaller than this of TC1 because the front is more stiff as illustrated by the top part of Fig. 10. As for TC1, the profiles corresponding to the extreme values of $s_{\mathrm{f}}$ are almost identical that those for $x_{\mathrm{f}}$. The isolines of the joint pdf highlight again the dissymmetry of the distribution.
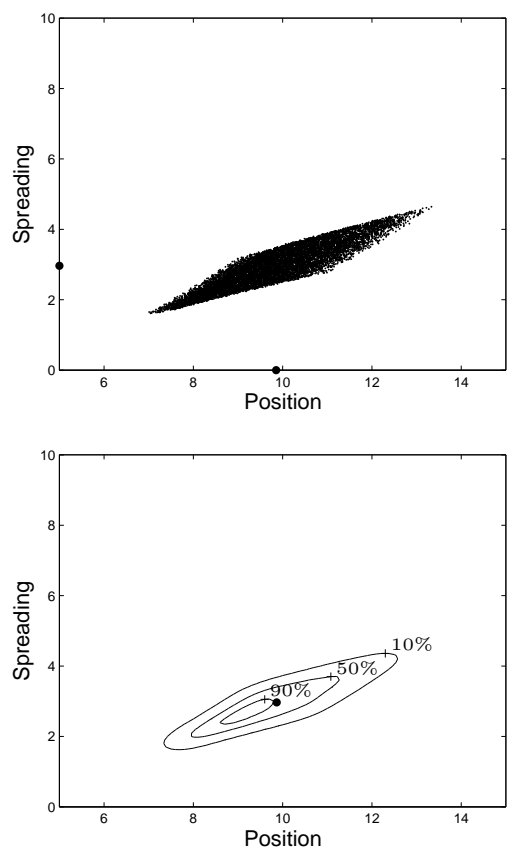

Figure 9: TC2 - top: $10^{4} \mathrm{PC}-\mathbb{P}_{2}$ realizations of $\left(x_{\mathrm{f}}, s_{\mathrm{f}}\right)$; bottom: isolines of the joint pdf.

\subsubsection{Effect of soil saturation}

We study the effect of the soil saturation state on the wetting front by considering five couples $\left(\psi_{\mathrm{b}}, \psi_{\mathrm{t}}\right)$ with constant difference $\psi_{\mathrm{b}}-\psi_{\mathrm{t}}=-9.25 \mathrm{~m}$. The computations are performed for $\mathrm{PC}-\mathbb{P}_{2}$. The first moments are reported in Tab. 4. As previously, increasing the initial saturation $\left(\psi^{0}\right)$ leads to higher mean front position, in agreement with the Darcy's law, but unlike TC1 no impact of the saturation on the mean spread is observed. This is due to the shape of the front which remains stiff for all the soil saturation considered. This is clearly illustrated on the bottom plot of Fig. 10. Another difference with TC1 is the large changes in the position standard deviation $\sigma\left(x_{\mathrm{f}}\right)$ for the five saturations. This sensitivity of the variability in $x_{\mathrm{f}}$ depending on the saturation causes a drastic change in the correlation between $x_{\mathrm{f}}$ and $s_{\mathrm{f}}$. Specifically, the coefficients of determination goes to zero as the saturation increases, as reported in the last column of Tab. 4] and illustrated in the scattered plots shown in Fig. 11, As for TC1, the uncertainty is amplified by the model, the coefficients of variation of the outputs being greater than those of the inputs. 

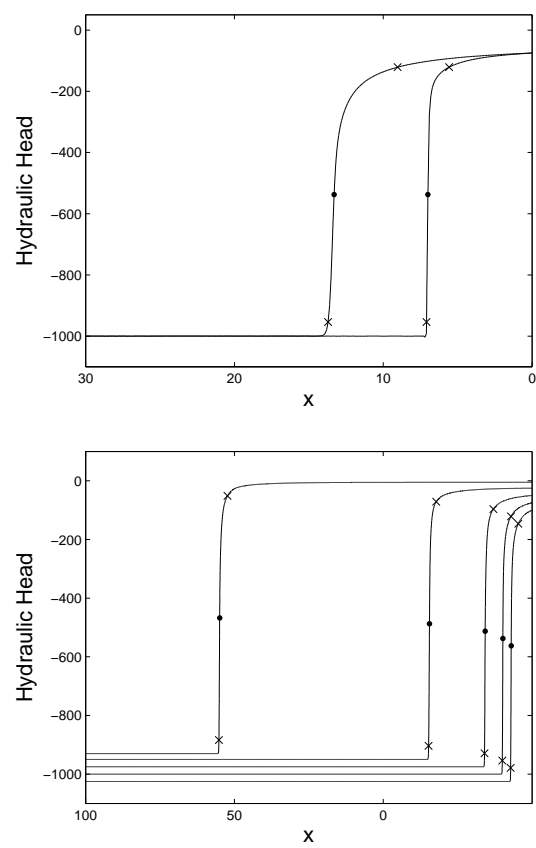

Figure 10: TC2 - top: Pressure corresponding to the extreme values of $x_{\mathrm{f}}$; bottom: Pressure obtained with $\overline{\boldsymbol{d}}$ for the five saturation states reported in Tab 4 .

Table 4: TC2 - Moments of the quantities of interest using $\mathrm{PC}-\mathbb{P}_{2}$ for five saturation states.

\begin{tabular}{ccccccccc}
\hline & & $x_{\mathrm{f}}$ & & \multicolumn{3}{c}{$s_{\mathrm{f}}$} & & \\
$\psi^{0}$ & $\psi_{\mathrm{t}}$ & $\mu$ & $\sigma$ & $c_{v}$ & $\mu$ & $\sigma$ & $c_{v}$ & $R^{2}$ \\
$c m$ & $c m$ & $c m$ & $c m$ & $\%$ & $c m$ & $c m$ & $\%$ & $\%$ \\
\hline-1025 & -100 & 7.09 & 0.91 & 13 & 2.62 & 0.59 & 23 & 85 \\
-1000 & -75 & 9.87 & 1.20 & 12 & 2.96 & 0.61 & 21 & 83 \\
-975 & -50 & 15.83 & 1.65 & 10 & 3.06 & 0.60 & 20 & 50 \\
-950 & -25 & 34.62 & 3.60 & 10 & 2.63 & 0.48 & 18 & 1 \\
-930 & -5 & 104.63 & 8.96 & 9 & 2.90 & 0.31 & 11 & 2 \\
\hline
\end{tabular}

First order sensitivity indices are plotted in Fig.12. Again, the two quantities of interest are found to be essentially additive models with low parameter interactions. For the position, the more influent parameter is $\epsilon$ in all cases except for the more saturated one where $\beta$ becomes dominant. For the spreading, on the contrary, the exponent $\beta$ is predominant, except for the most saturated configuration where $\epsilon$ takes over. The sensitivity analysis also reveals that the conductivity at saturation has only a weak impact of the front variability. Moreover, additional analysis for a deterministic water content law using $\theta(\psi, \overline{\boldsymbol{d}})$ and random conductivity has shown that the uncertainty in $K(\psi, \boldsymbol{d}(\boldsymbol{\xi}))$ is here responsible for essentially all the variability in the quantities of interest.

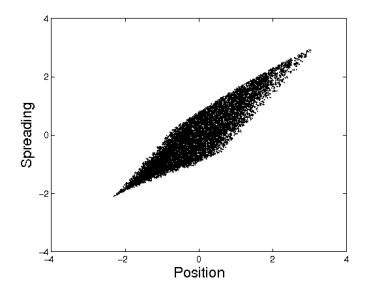

(a) $(-1025,-100)$

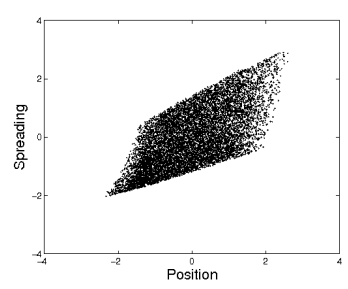

(b) $(-975,-50)$

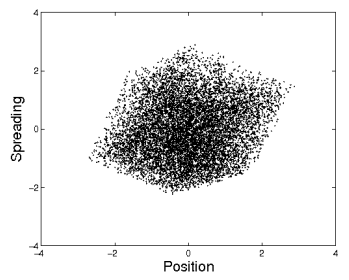

(c) $(-930,-5)$

Figure 11: TC2 - $10^{4} \mathrm{PC}-\mathbb{P}_{2}$ realizations of $\left(x_{\mathrm{f}}, s_{\mathrm{f}}\right)$ for three initial saturations. The realizations are centered and normalized.
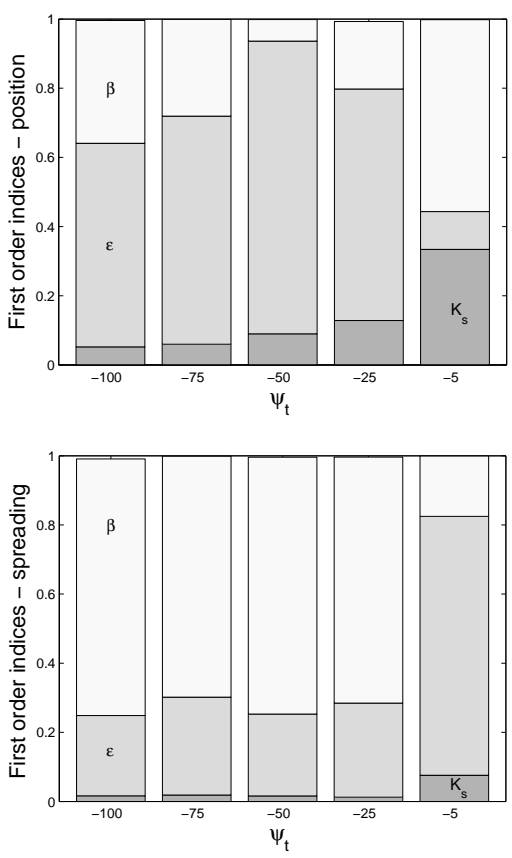

Figure 12: TC2 - First order sensitivity indices using PC - $\mathbb{P}_{2}$ for five saturation states.

\subsection{Test case 3}

\subsubsection{Test case description}

The third TC was proposed by Baca et al. (1997) [6]. The soil corresponds to a tilled layer modeled by (3),

$$
\theta(\psi, \boldsymbol{d}(\boldsymbol{\xi}))= \begin{cases}\theta_{\mathrm{s}}\left(\frac{\psi}{\psi_{\mathrm{a}}\left(\xi_{2}\right)}\right)^{-\frac{1}{\bar{b}}} & \text { if } \psi<\psi_{\mathrm{a}}\left(\xi_{2}\right), \\ \theta_{\mathrm{s}} & \text { if } \psi \geq \psi_{\mathrm{a}}\left(\xi_{2}\right)\end{cases}
$$


and

$$
K(\psi, \boldsymbol{d}(\boldsymbol{\xi}))= \begin{cases}K_{\mathrm{s}}\left(\xi_{1}\right)\left(\frac{\psi}{\psi_{\mathrm{a}}\left(\xi_{2}\right)}\right)^{-\gamma\left(\xi_{3}\right)} & \text { if } \psi<\psi_{\mathrm{a}}\left(\xi_{2}\right), \\ K_{\mathrm{s}}\left(\xi_{1}\right) & \text { if } \psi \geq \psi_{\mathrm{a}}\left(\xi_{2}\right) .\end{cases}
$$

The deterministic parameter is $\theta_{\mathrm{s}}=0.562$. The random parameters vector $\boldsymbol{d}=\left(K_{\mathrm{s}}, \psi_{\mathrm{a}}, \gamma\right)$ has components $\boldsymbol{d}_{i}$ uniformly distributed with $\pm 10 \%$ range and respective mean values $\overline{K_{\mathrm{s}}}=3.87 \cdot 10^{-4} \mathrm{~cm} . \mathrm{s}^{-1}, \overline{\psi_{\mathrm{a}}}=-4.55 \mathrm{~cm}$ and $\bar{\gamma}=2.23$. It corresponds to the ranges of variations

$$
K_{\mathrm{s}} \in 10^{-4} \cdot[3.48,4.26], \quad \psi_{\mathrm{a}} \in[-5,-4.1], \quad \gamma \in[2,2.45] .
$$

Furthermore, the exponent $\bar{b}$ is taken constant and equal to $3(\bar{\gamma}-2)^{-1}$, assuming a fixed pore-size distribution. For this choice, the variability of the water content depends only on $\psi_{\mathrm{a}}$, so the analysis focuses mainly on the uncertainties in the conductivity law $K$ as illustrated on Fig. 13. The length of the domain is $L=50 \mathrm{~cm}$, the final time is $T=1 h$, and the boundary and initial conditions are $\psi_{\mathrm{b}}=-1 m, \psi_{\mathrm{t}}=0, \psi^{0}(x)=\psi_{\mathrm{b}}$. Preliminary simulations have shown satisfying convergence of the deterministic discrete approximation for a mesh size $h=0.5 \mathrm{~cm}$ and a time steps $\delta t=2 \mathrm{~min}$. These discretization parameters are kept fixed in subsequent simulations.
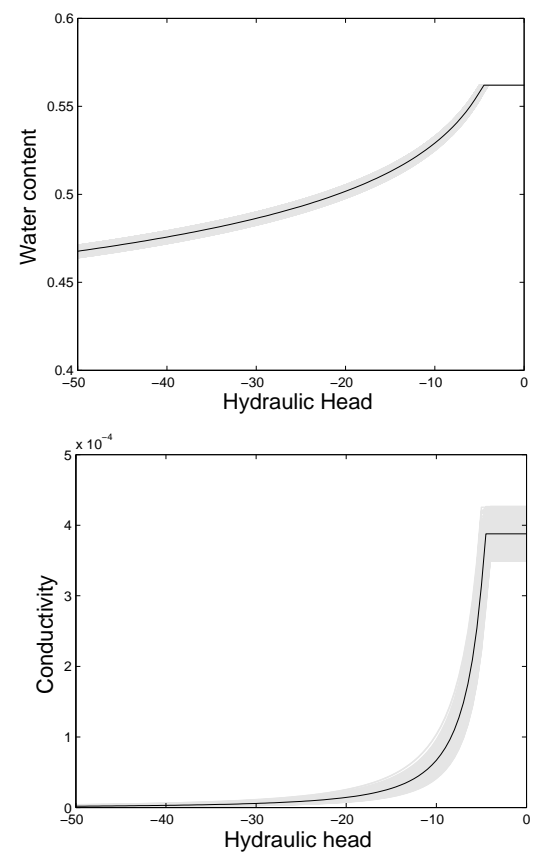

Figure 13: TC3 - Soil properties obtained for $\overline{\boldsymbol{d}}$ (black) and 500 random samples of $\boldsymbol{d}$ (gray).

\subsubsection{Validation of $P C E$ and first moments analysis}

Tab. 5 reports the first moments computed by MC using three sample sets of increasing dimension and PCE up to $\mathbb{P}_{3}$. The two approaches agree for sufficiently large sample set dimension (MC) and PCE order. MC requires a minimum of $10^{3}$ realizations while $\mathrm{PCE}$ requires less than 40 simulations. As for $\mathrm{TC} 1$ and $\mathrm{TC} 2$, the $\mathrm{PCE}$ requires a polynomial degree equal to 2 or 3 to correctly estimate the first three moments. As for TC2, a larger cubature formula level is required to ensure decay of $e$ (not shown). The detailed comparison with $\mathrm{MC}$ reveals that $\mathrm{PC}-\mathbb{P}_{2}$ provides a well-converged approximation, so this order is used in the following of this section.

Table 5: TC3 - moments and normalized error of the quan-

\begin{tabular}{|c|c|c|c|c|c|c|c|c|c|}
\hline & & $x_{\mathrm{f}}$ & & & & $s_{\mathrm{f}}$ & & & \\
\hline & $N_{\mathrm{sim}}$ & $\mu$ & $\sigma$ & $s$ & $e$ & $\mu$ & $\sigma$ & $s$ & $e$ \\
\hline & - & $\mathrm{cm}$ & $\mathrm{cm}$ & $\mathrm{cm}$ & $\%$ & $\mathrm{~cm}$ & $\mathrm{~cm}$ & $\mathrm{~cm}$ & $\%$ \\
\hline $\mathrm{MC}$ & $10^{2}$ & 22.27 & 1.09 & 0.37 & & 6.35 & 1.35 & 0.31 & \\
\hline $\mathrm{MC}$ & $10^{3}$ & 22.36 & 1.15 & 0.05 & & 6.41 & 1.25 & 0.32 & \\
\hline $\mathrm{MC}$ & $10^{4}$ & 22.33 & 1.16 & 0.08 & & 6.40 & 1.28 & 0.32 & \\
\hline $\mathrm{PC}-\mathbb{P}_{1}$ & 7 & 22.32 & 1.16 & 0.00 & 0.18 & 6.41 & 1.26 & 0.00 & 2.17 \\
\hline $\mathrm{PC}-\mathbb{P}_{2}$ & 19 & 22.32 & 1.16 & 0.07 & 0.05 & 6.39 & 1.29 & 0.34 & 0.63 \\
\hline $\mathrm{PC}-\mathbb{P}_{3}$ & 39 & 22.32 & 1.16 & 0.05 & 0.07 & 6.39 & 1.30 & 0.29 & 0.93 \\
\hline
\end{tabular}
tities of interest $x_{\mathrm{f}}$ and $s_{\mathrm{f}}$.
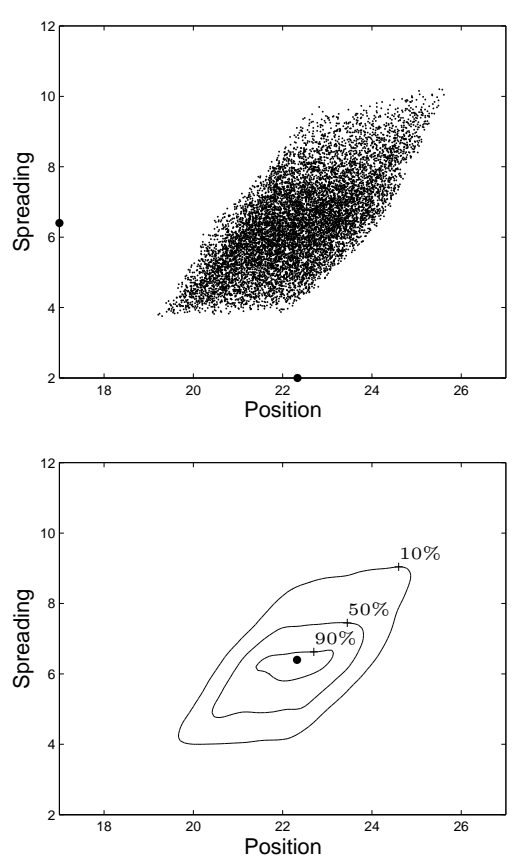

Figure 14: TC3 - top: $10^{4} \mathrm{PC}-\mathbb{P}_{2}$ realizations of $\left(x_{\mathrm{f}}, s_{\mathrm{f}}\right)$; bottom: isolines of the joint pdf.

Fig. 14 presents $10^{4}$ realizations of the the $\mathrm{PC}-\mathbb{P}_{2}$ couples $\left(x_{\mathrm{f}}, s_{\mathrm{f}}\right)$ with three isolines of the joint pdf. As previously, the scatter indicates that the more the velocity front increases, the more the front spreads as illustrated on the top plot of Fig. 15. Again, the position and the spreading are significantly linearly correlated with a correlation coefficient equal to $0.69\left(R^{2}=48 \%\right)$. 

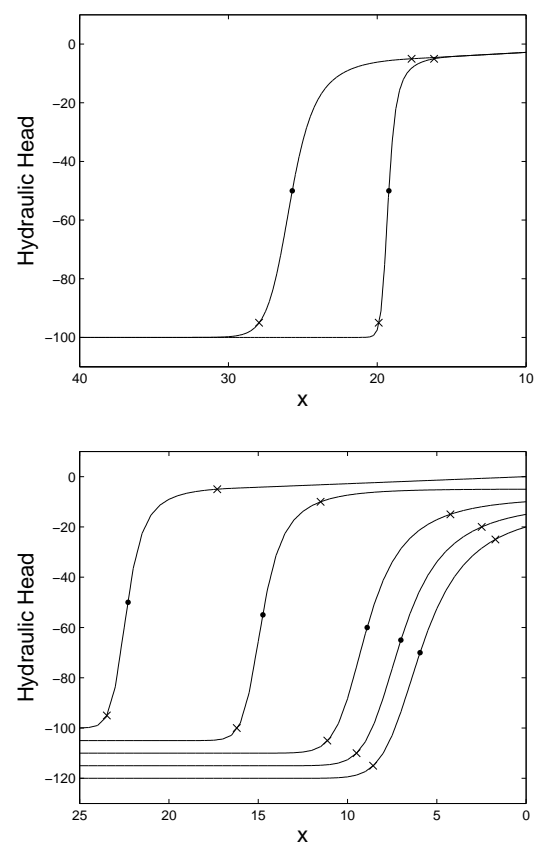

Figure 15: TC3 - top: Pressure corresponding to the extreme values of $x_{\mathrm{f}}$; bottom: Pressure obtained with $\overline{\boldsymbol{d}}$ for the five saturation states reported in Tab 6.

\subsubsection{Effect of soil saturation}

As previously, the impact of the initial saturation is analyzed by testing five couples $\left(\psi_{\mathrm{b}}, \psi_{\mathrm{t}}\right)$ with constant difference $\psi_{\mathrm{b}}-\psi_{\mathrm{t}}=-1 \mathrm{~m}$. The first moments computed from PC $-\mathbb{P}_{2}$ are reported in Tab. 6. As for TC1 and TC2, the mean of the position increases with the saturation. The bottom plot of Fig. 15 shows that the shape of the front for the average parameters values is not affected by the soil saturation. This explains that increasing the top boundary condition weakly impact the mean of the spreading comparing to TC1. Regarding standard deviations of the front position, it is found that the variability is weakly impacted by the saturation in contrast with TC2. As previously, an amplification of the input uncertainty is observed. At last, the coefficients of determination reveal a significant correlation between the position and the spreading, which tends to decay with the initial saturation, as for TC2, except for $\psi^{\mathrm{t}}=-100$ where the threshold head $\psi_{\mathrm{a}}$ is reached.

The first order sensitivity indices are plotted on Fig. 16. For the position, when the saturation is increased, the more influent parameter depends on the soil saturation and is successively $\gamma, \psi_{\mathrm{a}}$ and $K_{\mathrm{s}}$ with $S_{\gamma}^{x_{\mathrm{f}}}(-10)=0.63$, $S_{\psi_{\mathrm{a}}}^{x_{\mathrm{f}}}(-5)=0.66$ and $S_{\psi_{\mathrm{s}}}^{x_{\mathrm{f}}}(0)=0.57$. For the spreading, the exponent is always the most influent although for the most saturated state the second influent parameter becomes significant with $S_{\psi_{a}}^{s_{\mathrm{f}}}(0)=0.47$, due to threshold effect as explain previously. Again, the influence of the conductivity at saturation increases with the saturation for the position and is negligible for the spreading.
Table 6: TC3 - Moments of the quantities of interest using $\mathrm{PC}-\mathbb{P}_{2}$ for five saturation states.

\begin{tabular}{ccccccccc}
\hline & & $x_{\mathrm{f}}$ & \multicolumn{7}{c}{$s_{\mathrm{f}}$} \\
$\psi^{0}$ & $\psi_{\mathrm{t}}$ & $\mu$ & $\sigma$ & $c_{v}$ & $\mu$ & $\sigma$ & $c_{v}$ & $R^{2}$ \\
$\mathrm{~cm}$ & $c m$ & $c m$ & $c m$ & $\%$ & $c m$ & $c m$ & $\%$ & $\%$ \\
\hline-120 & -20 & 5.99 & 0.90 & 15 & 6.97 & 1.35 & 19 & 98 \\
-115 & -15 & 7.06 & 0.99 & 14 & 7.14 & 1.36 & 19 & 96 \\
-110 & -10 & 8.95 & 1.12 & 12 & 7.00 & 1.31 & 19 & 89 \\
-105 & -5 & 14.81 & 1.54 & 10 & 4.82 & 1.04 & 22 & 28 \\
-100 & 0 & 22.32 & 1.16 & 5 & 6.39 & 1.29 & 20 & 49 \\
\hline
\end{tabular}
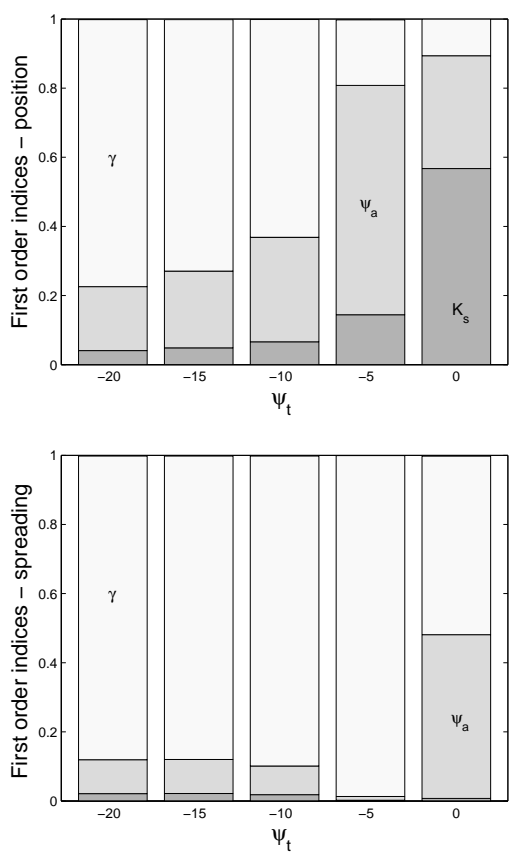

Figure 16: TC3 - First order sensitivity indices using PC - $\mathbb{P}_{2}$ for five saturation states.

\subsection{Effect of saturated conductivity distribution}

The conclusion regarding the respective influence of the model parameters depends on the assumed distribution. To illustrate this aspect we repeat the analyses above but for a saturated conductivity having a log-uniform distribution with higher variability than previously, the distributions of the other parameters being kept constant. Specifically, we assume for $K_{\mathrm{s}}$ a log-uniform distribution over one decade. The parametrization is expressed as

$$
K_{\mathrm{S}}(\xi)=e^{\ln (A)+\xi(\ln (B)-\ln (A))}, \quad \xi \sim \mathcal{U}[0,1],
$$

with $[A, B]=2.5 \cdot\left[10^{-3}, 10^{-2}\right]$ for TC1 and TC2,

$$
[A, B]=\left[10^{-4}, 10^{-3}\right] \text { for TC3. }
$$

The bounds $A$ and $B$ of the uncertainty ranges are selected such that the mean values of $K_{\mathrm{s}}$ are essentially the same as for the previous uniform distributions for the TC. However, the variability in the $K_{\mathrm{s}}$ are significantly increased 
for the log-uniform distributions, with coefficients of variation roughly equal to $64 \%$ compared to roughly $6 \%$ in the uniform case.

As previously, a satisfactory convergence is achieved for the second-order expansion $\mathrm{PC}-\mathbb{P}_{2}$ (not shown), for which the estimated the first moments of the outputs are reported and compared with the case of the uniform distribution (bracketed values) in Table 7. The table shows that the expected values of the outputs are weakly (TC1 and TC3) to moderately (TC2) affected by the changes in the distributions of $K_{\mathrm{s}}$. On the contrary, the standard deviations and the skewness of the outputs are larger for the log-uniform case, as one may have anticipated from the larger variability in the conductivity at saturation. This can be better appreciated from the outputs pdf's which are reported for the two distributions in Fig. 17 for TC1 (cases of TC2 and TC3, not shown, are similar).

Concerning the sensitivity analysis, Table 8 compares the relative influence of the parameters for the uniform and log-uniform distributions for $K_{\mathrm{s}}$ and each TC. It is seen that the increase in the variability of $K_{\mathrm{s}}$ yields a drastically larger relative influence for the conductivity, as measured by the first order sensitivity indices, which is now the dominant source of uncertainty for all the TC. In fact, if the log-uniform distribution had been defined to match both the mean and standard deviation of the uniform case, the conclusion of the sensitivity analysis would have remained essentially the same as for the uniform distribution (not shown). This exercise highlights the importance of the assumed prior distribution of the parameters on the conclusions drawn from the sensitivity analysis.

Table 7: Moments of the quantities of interest using PC - $\mathbb{P}_{2}$ for a log-uniform distribution of $K_{\mathrm{s}}$. Results with a uniform distribution of $K_{\mathrm{s}}$ are in bracket.

\begin{tabular}{ccccccc}
\hline & $x_{\mathrm{f}}$ & & \multicolumn{3}{c}{$s_{\mathrm{f}}$} \\
& $\mu$ & $\sigma$ & $s$ & $\mu$ & $\sigma$ & $s$ \\
& $c m$ & $c m$ & $c m$ & $c m$ & $c m$ & $c m$ \\
\hline \multirow{2}{*}{$\mathrm{TC} 1$} & 22.33 & 10.65 & 0.74 & 14.08 & 4.24 & 0.54 \\
& {$[22.33]$} & {$[3.27]$} & {$[0.20]$} & {$[15.14]$} & {$[3.10]$} & {$[0.47]$} \\
\hline \multirow{2}{*}{$\mathrm{TC} 2$} & 6.99 & 2.49 & 0.51 & 2.32 & 0.90 & 0.61 \\
& {$[9.87]$} & {$[1.21]$} & {$[0.18]$} & {$[2.96]$} & {$[0.61]$} & {$[0.16]$} \\
\hline \multirow{2}{*}{$\mathrm{TC} 3$} & 21.82 & 9.70 & 0.59 & 6.08 & 1.77 & 0.59 \\
& {$[22.32]$} & {$[1.16]$} & {$[0.07]$} & {$[6.39]$} & {$[1.29]$} & {$[0.34]$} \\
\hline
\end{tabular}

\section{Conclusion}

In this work, we have studied the impact of the variability in soil properties on subsurface flows governed by Richards' equation. We have considered random parameters in the water content and the conductivity constitutive equations for three common models of the literature, namely the Haverkamp's, the Van Genuchten's and the Brooks-Corey's relations. An accurate second order
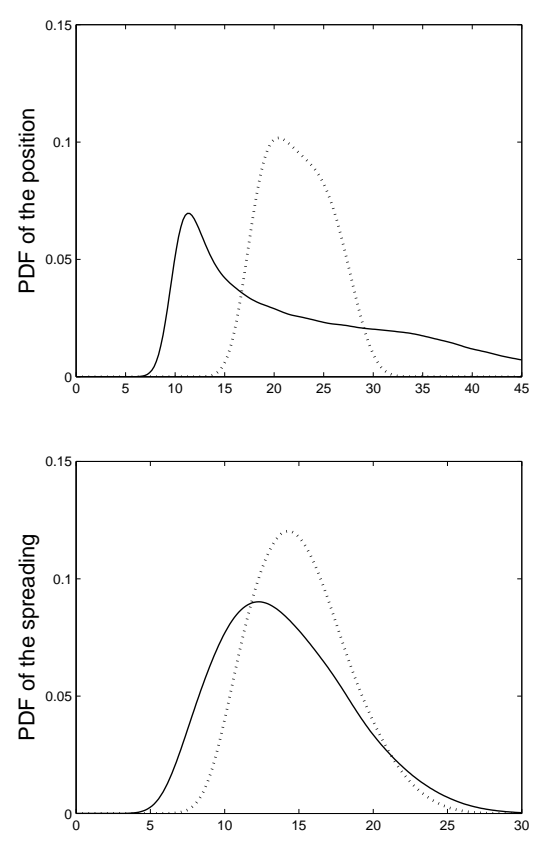

Figure 17: TC1: Pdf's of outputs with uniform (dotted line) and log-uniform (solid line) distributions of $K_{\mathrm{s}}$.

Table 8: First order sensitivity indices using $\mathrm{PC}-\mathbb{P}_{2}$ for a log-uniform distribution of $K_{\mathrm{s}}$. Results with a uniform distribution of $K_{\mathrm{s}}$ are in bracket.

\begin{tabular}{cccc}
\hline & & $x_{\mathrm{f}}$ & $s_{\mathrm{f}}$ \\
\hline & $K_{\mathrm{s}}$ & $0.89[0.07]$ & $0.52[0.00]$ \\
& $A$ & $0.08[0.84]$ & $0.20[0.45]$ \\
$\mathrm{TC} 1$ & $\gamma$ & $0.00[0.05]$ & $0.19[0.42]$ \\
& $\alpha$ & $0.00[0.01]$ & $0.05[0.11]$ \\
& $\beta$ & $0.00[0.03]$ & $0.00[0.00]$ \\
\hline & $K_{\mathrm{s}}$ & $0.90[0.06]$ & $0.67[0.02]$ \\
$\mathrm{TC} 2$ & $\epsilon$ & $0.08[0.66]$ & $0.07[0.27]$ \\
& $\beta$ & $0.01[0.28]$ & $0.22[0.70]$ \\
\hline & $K_{\mathrm{s}}$ & $0.99[0.57]$ & $0.51[0.00]$ \\
$\mathrm{TC} 3$ & $\psi_{\mathrm{a}}$ & $0.00[0.33]$ & $0.22[0.47]$ \\
& $\gamma$ & $0.00[0.10]$ & $0.24[0.52]$ \\
\hline
\end{tabular}

discontinuous Galerkin method has been employed to discretize the deterministic problem in space and time. To describe the impact of uncertainties, Polynomial Chaos expansions of the model outputs were constructed by means of a non-intrusive spectral projection. The output quantities we focused on are the position and the spreading of the wetting front. For each hydrological laws, the effect of the initial saturation state was investigated.

The results, and validation with Monte-Carlo simulations, demonstrate that low order Polynomial Chaos expansions are well-adapted to represent our quantities of interest. Specifically, for the three test cases, a second order expansion provides accurate estimations of first output moments and probability density functions. The computation of the output Polynomial Chaos expansions requires 
a number of simulations lower by two orders of magnitude compared to Monte-Carlo. This is due to the smooth mapping between the input parameters and the model outputs considered in this work, which allows for accurate low order polynomial approximations to be efficiently computed from a limited number of model simulations. However, this may not be always the case. In particular, other model outputs may present much more complex dependences requiring significantly higher polynomial degrees to be accurately approximated. In such situations, preconditioning of the output to be projected may be an effective alternative to recover a fast convergence rate with respect to the polynomial degree, see Alexanderian et al. $([2,[3])$. The worst situation corresponds to outputs which present singularities or discontinuities with respect to the model parameters (i.e. parametric bifurcations), inducing a slow convergence of spectral polynomial approximations. Here, local approximations based on piecewise polynomial representations over $\Xi$ should be employed, for instance introducing stochastic multi-wavelet decompositions, see Le Maître et al. [33, 34]. Finally, the extension of the methodology to model parameters with values varying in space (random fields), if conceptually immediate, will raise the issue of the dimensionality of the parameter space. In general, to limit the increasing computational cost of the NISP method with the number of uncertain parameters, the introduction of adaptive constructions for the cubature rule, following for instance Gerstner and Griebel [25], will be a key ingredient to maintain efficiency.

Analysis of the output variability yields a first conclusion that the advection and the diffusion of the wetting front are well-correlated for all models and most saturation states. Two others important findings of this work are that the three models increase the input uncertainty and that the variability of the front diffusion depends on the saturation state. Results of the global sensitivity analysis can be summarized in three main conclusions. First, for each model the more influent parameter depends on the soil saturation state. Second, the interaction between the input parameters are negligible for all models. Lastly, the influence of the saturated conductivity increases with the soil saturation for the position but is minor for the spreading.

The sensitivity analyses presented for the different models are clearly of an a priori nature since the conclusions depend on the assumed probability distributions of the relevant parameters in the models. This was highlighted by considering higher variability in the $K_{s}$ with log-uniform distribution, resulting in a larger relative influence of this parameter. This exercise shows that expertise, or measurements, are needed for a fair assessment of the respective parameters influence. Similarly, our simulations assumed the statistical independence of the input parameters, while experimental evidences may reveal correlations. In fact, the effects of correlation and dependencies between input parameters can readily be investigated a priori using the constructed PC model (based on the independence as- sumption). Indeed, the PC model being convergent in the mean square sense, it can be resampled at will for other probability distributions whose supports are included in the support of the distribution used to generate the PC expansion, without having to rely on additional full-model solution. This is another interest in using the PC expansions that we plan to exploit in the future. Finally, a pure Monte-Carlo sensitivity analysis could have been performed for the present simple situation (and was in fact used for validation and error computations), owing to the low computational cost of the model solves; however the advantages of the PC approach are expected to become more significant when considering domains in higher dimensions and more complex parameter distributions, in particular for random fields based on experimental characterization of soils properties, for which the Monte-Carlo methods can become prohibitively costly. Current works are focusing on this aspect.

\section{Acknowledgement}

The work of OLM is partially supported by the King Abdullah University of Science and Technology through the SRI-center on Uncertainty Quantification in Computational Science and Engineering.

\section{Appendix A. Smolyak formula and cubature rule}

We provide a brief overview of the Smolyak formula for the construction of sparse integration formula in $d$ dimensions. Consider a nested sequence of one-dimensional quadrature formulas,

$$
\int f d x \approx I^{l} f \doteq \sum_{i=1}^{q_{l}} f\left(x_{i}^{l}\right) \omega_{i}^{l}, \quad l=1,2, \ldots
$$

where $l$ denotes the level of the formula. The nested character implies that the quadrature points $\left\{x_{i}^{l}, i=1, \ldots, q_{l}\right\}$ belongs to the set of points of formulas with level $l^{\prime} \geq l$. Typically, the number of points at a level $l$ is $q_{l} \sim 2^{l}$. The multi-dimensional formula at level $l$ for the full tensorization is

$$
\begin{aligned}
& \int \ldots \int f\left(x_{1}, \ldots, x_{d}\right) d x_{1} \ldots d x_{d} \approx I^{l} \otimes \cdots \otimes I^{l} f \\
&=\sum_{i_{1}=1}^{q_{l}} \ldots \sum_{i_{d}=1}^{q_{l}} f\left(x_{i_{1}}^{l}, \ldots x_{i_{d}}^{l}\right) w_{i_{1}}^{l} \ldots w_{i_{d}}^{l} .
\end{aligned}
$$

Introducing the one-dimensional difference formulas,

$$
\delta I^{l} f=\left(I^{l}-I^{l-1}\right) f, \quad \delta I^{1}=I^{1}
$$

and the mult-index $\boldsymbol{l}=\left(l_{1} \ldots, l_{d}\right) \in \mathbb{N}^{d}$, the tensored formula can be expressed as

$$
I^{l} \otimes \cdots \otimes I^{l} f=\sum_{|\boldsymbol{l}|_{\infty} \leq l} \delta^{l_{1}} \otimes \cdots \otimes \delta^{l_{d}} f, \quad|\boldsymbol{l}|_{\infty}=\max _{i} l_{i}
$$


The level $l$ Smolyak sparse tensorization, see (Smolyak, 1963 [4] ), is obtained by restricting the summation of the tensored differences to the reduced set of multi-indexes as follows

$$
\int \ldots \int f\left(x_{1}, \ldots, x_{d}\right) d x_{1} \ldots d x_{d} \approx \sum_{|l| \leq l+d-1} \delta^{l_{1}} \otimes \cdots \otimes \delta^{l_{d}} f
$$

where $|\boldsymbol{l}|=\sum_{i} l_{i}$. The left-hand side of the previous equation can be recast as a cubature rule

$$
\int \ldots \int f\left(x_{1}, \ldots, x_{d}\right) d x_{1} \ldots d x_{d} \approx \sum_{j=1}^{N_{l}} w_{j} f\left(\boldsymbol{x}_{j}\right)
$$

where the total number of cubature points depends on the level $l$. The cubature points are plotted in Fig. A.18 in the case $d=3$ and $l=4$. The sparse tensorization yields a significantly lower number of cubature points, compared to the fully tensored formula, and one can easily determined the polynomial functions $f$ which are exactly integrated from the properties of the underlying one-dimensional sequence. In this work, we rely on cubature rules based on nested Konrod-Patterson formulas (Petras, 2000 [40] and 2001 [41]) and (Keese and Matthies, 2003 [29]) for integration over hypercubes.

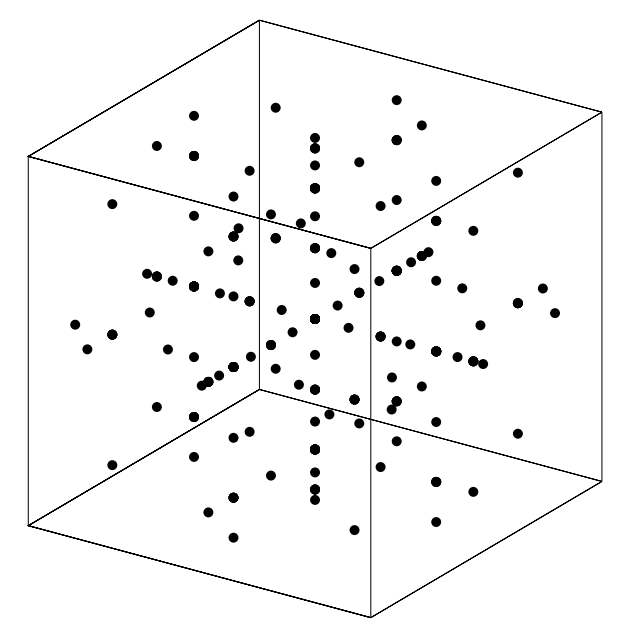

Figure A.18: Cubature points for the numerical integration over $[0,1]^{3}$ and level $l=4$.

\section{References}

[1] Abramowitz, M., Stegun, I.A.: Handbook of Mathematical Functions, Dover, (1970)

[2] Alexanderian, A., Le Maître, O.P., Najm, H.N., Iskandarani, M., Knio, O.M.: Multiscale stochastic preconditionners in non-intrusive spectral projection, SIAM J. Sci. Comp., 50:7, 306-340, (2012)

[3] Alexanderian, A., Rizzi, F., Rathinam, M., Le Maître, O.P., Knio, O.M.: Preconditioned Bayesian Regression for Stochastic Chemical Kinetics, J. Sci. Comp., in press, (2013)
[4] Arnold, D.N. , Brezzi, F., Cockburn, B., Marini, L.D.: Unified analysis of discontinuous Galerkin methods for elliptic problems, SIAM J. Numer. Anal., 39(5), 1749-1779, (2001)

[5] Assouline, A., Tartakovsky, D.M.: Unsaturated hydraulic conductivity function based on a soil fragmentation process, Water Resour. Res., 37(5), 1309-1312, (2001)

[6] Baca, R.G., Chung, J.N., Mulla, D.J.: Mixed transform finite element method for solving the nonlinear equation for flow in variably saturated porous media, Int. J. Numer. Meth. Fl., 24, 441-455, (1997)

[7] Bastian, P.: Higher order discontinuous Galerkin methods for flow and transport in porous media, Challenges in scientific computing-CISC 2002, Lect. Notes Comput. Sci. Eng., 35, 1-22, Springer, Berlin, (2003)

[8] Bastian, P., Ippisch, O., Rezanezhad, F., Vogel, H.J., Roth, K.: Numerical simulation and experimental studies of unsaturated water flow in heterogeneous systems, Reactive flows, diffusion and transport, 579-597, Springer, Berlin, (2007)

[9] Besbes, M., De Marsily, G.: From infiltration to recharge: use of a parametric transfer function, J. Hydrol., 74, 271-293, (1984)

[10] Bowman, A.W., Azzalini, A.: Applied smoothing techniques for data analysis, New York: Oxford University Press, (1997)

[11] Bresler, B., Dagan, G.: Unsaturated flow in spatially variable fields.2. Application of water flow models to various fields, Water Resour. Res., 19, 413-420, (1983)

[12] Brooks, R.H., Corey, A.T.: Hydraulic properties of porous media, Hydrology paper, Fort Collins, Colorado: Colorado State University, 3:27, (1964)

[13] Cameron , R.H., Martin, W.T.: The orthogonal development of nonlinear functionals in series of Fourier-Hermite functionals, Ann. Math., 48, 385392, (1947)

[14] Celia, M.A., Bouloutas, E.T.: A General MassConservative Numerical Solution for the Unsaturated Flow Equation, Water Resour. Res., 26(7), 1483-1496, (1990)

[15] Cockburn, B., Shu, C.-W.: The local discontinuous Galerkin method for time-dependent convectiondiffusion systems, SIAM J. Numer. Anal., 35(6), 2440-2463, (1998)

[16] Crestaux, T., Le Maître, O, Martinez, J.M.: Polynomial Chaos Expansion for Sensitivity Analysis, Reliab. Eng. and Syst. Saf., 94(7), 1161-1172, (2009)

[17] Curtiss, C.F., Hirschfelder, J.O.: Integration of stiff equations, Proc. Nat. Acad. Sci. U.S.A., 38, 235243, (1952)

[18] Dillah, D.D., Protopapas, A.L.: Uncertainty propagation in layered unsaturated soils, Transport porous med., 38, 273-290, (2000)

[19] Dostert, P., Efendiev, Y., Mohanty, B.: Efficient uncertainty quantification techniques in inverse problems for Richards' equation using coarsescale simulation models, Adv. water resour., 32, 329 - 339, (2009)

[20] Fagherazzi, S., Furbish, D.J., Rasetarinera, P., Hussaini, M.Y.: Application of the discontinuous spectral Galerkin method to groundwater flow, Adv. water resour., 27, 129-140, (2004)

[21] Forster, R.: On the stochastic Richards equation, PhD thesis, Freie Universitaät Berlin, (2011)

[22] Gardner, W.R., : Some steady state solutions of unsaturated moisture flow equations with applica- 
tions to evaporation from a water table, Soil. Sci., 85, 228-232 (1958)

[23] Ghanem, R.G., Spanos, S.D.: Stochastic Finite Elements: a Spectral Approach, Springer Verlag, (1991)

[24] Green, W.H., Ampt, G.: Studies on soils physics,1. The flow of air and water through soils, J. Agric. Sci., 4(1), 1-24, (1911)

[25] Gerstner, T., Griebel, M. : Dimension- adaptive tensor-product quadrature, Computing, 71, 65-87, (2003)

[26] Haverkamp, R., Vauclin, M., Touma, J., Wierenga, P.J., Vachaud, G.: A Comparison of Numerical Simulation Models for one-dimensionnal Infiltration, Soil Sci. Soc. Am. J., 41, 285-294, (1977)

[27] Helton, J.C., Davis, F.J.: Latin Hypercube sampling and the propagation of uncertainty in analyses of complex systems., Reliab. Eng. and Syst. Saf., 81(1), 23-69, (2003)

[28] Homma, T., Saltelli, A.: Importance measures in global sensitivity analysis of nonlinear models, Reliab. Eng. and Syst. Saf., 52(1), 1-17, (1996)

[29] Keese, A., Matthies, H.G., Numerical methods and Smolyak quadrature for nonlinear stochastic partial differential equations, Institute of Scientific Computing TU Braunschweig Brunswick, (2003)

[30] Klieber, W., Rivière, B.: Adaptive simulations of two-phase flow by discontinuous Galerkin methods, Comput. Methods Appl. Mech. Engrg., 196(1-3), 404-419, (2006)

[31] Knabner, P., Schneid, E.: Adaptive Hybrid Mixed Finite Element Discretization of Instationary Variably Saturated Flow in Porous Media, High Perform. Sci. and Engrg. Comp., 29, 37-44, (2002)

[32] Kool, J.B., Parker, J.C., Van Genuchten, M.Th. Parameter estimation for unsaturated flow and transport models - a review, J. Hydrol., 91, 255293, (1987)

[33] Le Maître, O.P., Knio, O.M., Najm, H.N., Ghanem, R.G.: Uncertainty Propagation Using Wiener-Haar Expansions, J. Comp. Phys., (197):1, 28-57, (2004)

[34] Le Maître, O.P., Najm, H.N., Knio, O.M., Ghanem, R.G.: Multi-Resolution Analysis of Wiener-Type Uncertainty Propagation Schemes, J. Comp. Phys., (197):2, 502-531, (2004)

[35] Le Maître, O., Knio, O.M.: Spectral methods for uncertainty quantification, Springer, (2010)

[36] Lu, Z., Zhang, D.: On stochastic modeling of flow in multimodal heterogeneous formations, Water Resour. Res., 38(10), 1190, doi:10.1029/2001WR001026 (2002)

[37] Mantoglou, A., Gelhar, L.W.: Stochastic modeling of large-scale transient unsaturated flow systems, Water Resour. Res., 23(1), 37-46, (1987)

[38] Manzini, G., Ferraris, S.: Mass-conservative finite volume methods on 2-D unstructured grids for the Richards equation, Adv. water resour., 27, 11991215, (2004)

[39] Moskowitz, B., Caflish, R. E.: Smoothness and dimension reduction in Quasi-Monte Carlo methods, Math. Comp. Model., 23(8), 37-54, (1996)

[40] Petras, K., On the Smolyak Cubature Error for Analytic Functions, Advances in Computational Mathematics, 12, 71-93, (2000)

[41] Petras, K., Fast calculation of coefficients in the Smolyak algorithm, Num. Algo., 26, 93-109, (2001)

[42] Protopapas, A.L., Bras, R.L.: Uncertainty propagation with numerical models for flow and transport in the unsaturated zone, Water Resour. Res., 26(10), 2463-2474, (1990)

[43] Reagan, M.T., Najm, H.N., Ghanem, R.G., Knio,
O.M., Uncertainty quantification in reacting flow simulations through non-intrusive spectral projection, Combustion and Flames, 132, 545-555, (2003)

[44] Richards, L.A.: Capillary Conduction of liquids through porous mediums, Physics, 1, 318-333, (1931)

[45] Sacks, J., Welch, W.J., Mitchell, T.J., Wynn, H.P. : Design and analysis of computer experiments, Stat. Sci., 4(4), 409-435, (1989)

[46] Smolyak, S.A.: Quadrature and interpolation formulas for tensor products of certain classes of functions, Dokl. Akad. Nauk SSSR, 4, 240-243, (1963)

[47] Sobol, I.M.: Sensitivity estimates for nonlinear mathematical models, Math. Mod. and Comput. Exp., 1(4), 407-414, (1993)

[48] Sochala, P., Ern, A., Piperno, S.: Mass conservative BDF-discontinuous Galerkin/explicit finite volume schemes for coupling subsurface and overland flows, Comput. Methods Appl. Mech. Engrg., 198, 21222136, (2009)

[49] Tartakovsky, D. M., Guadagnini, A., Riva, M.: Stochastic averaging of nonlinear flows in heterogeneous porous media, Journal of Fluid Mechanics, 492, 9, 47-62, (2003)

[50] Tartakovsky, A. M., Garcia-Naranjo L., Tartakovsky, D. M.,: Transient flow in a heterogeneous vadose zone with uncertain parameters, Vadose zone journal, 3(1), 154-163, (2004)

[51] Van Genuchten, M.Th.: A Closed-form Equation for Predicting the Hydraulic Conductivity of Unsaturated Soils, Soil Sci. Soc. Am. J., 44(5), 891-898, (1980)

[52] Vogel, T., Van Genuchten, M.Th., Cislerova, M.: Effect of the shape of the soil hydraulic functions near saturation on variably-saturated flow predictions, Adv. water resour., 24, 133-144, (2001)

[53] Wang, P., Tartakovsky, D.M.: Reduced complexity models for probabilistic forecasting of infiltration rates, Adv. water resour., 34, 375-382, (2011)

[54] Woodward, C., Grant, K., Maxwell, R.: Applications of sensitivity analysis to uncertainty quantification in variably saturated flow, Computational Methods in Water Resources Proceedings of the XIVth International Conference on Computational Methods in Water Resources (CMWR XIV), 47, 73 $-80,(2002)$

[55] Xiu, D., Karniadakis, G.E.: The Wiener-Askey Polynomial Chaos for Stochastic Differential Equations, SIAM J. Sci. Comput., 24(2), 619-644, (2002) 